\title{
Riqueza, estrutura e interações edáficas em um gradiente de restinga do Parque Estadual do Acaraí, Estado de Santa Catarina, Brasil ${ }^{1}$
}

\author{
João Carlos Ferreira de Melo Júnior ${ }^{2,3}$ e Maria Regina Torres Boeger ${ }^{2}$
}

Recebido: 7.08.2014; aceito: 16.12.2014

\begin{abstract}
Richness, structure, and edaphic interactions in the restinga gradient of Parque Estadual do Acaraí, Santa Catarina State, Brazil). The present work characterized the flora and phytocommunities of a restinga gradient on the coast plain of Santa Catarina State and correlated them to edaphic attributes. The floristic survey followed the wide patrolling method and the community structure was determined through the plot method. Soil samples of each physiognomy were collected for analysis of macronutrients, organic matter, moisture, and litter thickness. Flora comprises 319 species, 244 of which occur exclusively in formations of herbaceous restinga (63), shrub restinga (32), shrub-tree restinga (46), and transition forest (103). The structural pattern of each restinga physiognomy studied was influenced by edaphic factors as organic matter, potassium, and aluminum contents, cation exchange capacity, and litter thickness. These attributes are determinant for the flora characterizing each phytocommunity.
\end{abstract}

Keywords: diversity, phytocommunities ecology, plant-soil relationships, soil gradient

RESUMO - (Riqueza, estrutura e interações edáficas em um gradiente de restinga do Parque Estadual do Acaraí, Estado de Santa Catarina, Brasil). O presente trabalho teve como objetivo caracterizar a flora e as fitocomunidades de um gradiente de restinga da planície litorânea Estado de Santa Catarina, correlacionando-as com os atributos edáficos. Para o levantamento florístico foi utilizado o wide patrolling, enquanto a estrutura da comunidade foi determinada pelo método de parcelas. Amostras de solo de cada fisionomia foram coletadas para análise de macronutrientes, matéria orgânica, umidade e espessura da serapilheira. A flora é composta por 319 espécies, dentre as quais 244 ocorrem de forma exclusiva nas formações de restinga herbácea (63), restinga arbustiva (32), restinga arbustivo-arbórea (46) e floresta de transição (103). O padrão estrutural de cada fisionomia da restinga estudada foi influenciado por fatores edáficos, como os teores de matéria orgânica, potássio, alumínio, a capacidade de troca catiônica e espessura da serapilheira, sendo estes atributos determinantes da flora característica de cada fitocomunidade.

Palavras-chave: diversidade, ecologia de fitocomunidades, gradiente edáfico, relações planta-solo

\section{Introdução}

Pertencente ao domínio da Mata Atlântica, as restingas são formadas por um mosaico de comunidades vegetais florística e fisionomicamente distintas e ocupam as planícies arenosas de origem fluvio-marinha de idade quaternária, localizadas na costa brasileira (Eiten 1983, Araujo \& Henriques 1984, Coutinho 2006, Magnano et al. 2010, IBGE 2012). Estão submetidas à condições ambientais extremas, caracterizadas por altas temperaturas, ventos constantes, elevada salinidade e deficiência em nutrientes (Scarano et al. 2001, Scarano 2002). Variações fisionômicas são observadas desde a região pós-praia até as áreas mais interiores da planície costeira (Veloso et al. 1991, Oliveira-Filho \& Carvalho 1993). As formações herbáceas, arbustivas e florestais constituem as principais fisionomias de restinga, baseadas na estrutura da vegetação (Silva \& Britez 2005). Sua diversidade biológica é proveniente do Cerrado, da Caatinga e de outros ecossistemas da Mata Atlântica, e representada por espécies aclimatadas à faixa litorânea (Araujo 2000). A riqueza florística e a diversidade funcional das restingas aumentam

1. Parte da Tese de Doutorado do primeiro Autor

2. Programa de Pós-Graduação em Ecologia e Conservação da Universidade Federal do Paraná, Caixa Postal 19031, CEP 81531-990 Curitiba, PR, Brasil

3. Autor para correspondência: jcmelo_wood@hotmail.com 
gradativamente das dunas em direção às formações florestais mais distantes do mar (Fernandes 2006).

As formações de restinga são definidas pela influência marinha e pelas condições de solo (Souza 2004, Sampaio et al. 2005). Os solos associados à região pós-praia possuem coloração amarela, pouca impregnação de ácido húmico, baixa disponibilidade de matéria orgânica e capacidade de retenção de água e nutrientes. Já os solos das formações arbóreas são superficialmente brancos, com maior teor de matéria orgânica e maior impregnação de ácidos húmicos (Araujo \& Lacerda 1987, Martin et al. 1997, Gomes et al. 2007, Almeida Jr. et al. 2009). Dessa maneira, as espécies das formações mais próximas ao mar são conhecidas por sobreviverem em condições severas e bastante limitadas em seu suprimento nutricional (Fernandes 2006).

As comunidades de restinga dependem das condições edáficas (Falkenberg 1999) e exercem uma importante ação sobre a estabilização do substrato nesse ambiente (Assumpção \& Nascimento 2000). Apesar dessa dependência, os principais estudos sobre os padrões de diversidade ao longo dos $5.000 \mathrm{~km}$ de extensão de restinga no litoral brasileiro (Holzer et al. 2004) focam, principalmente, nos levantamentos florísticos e fitossociológicos (Almeida Jr. et al. 2011).

Dos estudos realizados na região sul do Brasil, e que contribuíram para o conhecimento da diversidade florística ou da organização estrutural das comunidades de restinga, predominam os levantamentos realizados nos Estados do Rio Grande do Sul e do Paraná (Silva 1999). Para o Estado de Santa Catarina, há um menor registro de investigações sobre a vegetação costeira (Reitz 1954, Reitz 1961, Bresolin 1979, Cordazzo \& Costa 1989, Danilevicz et al. 1990, Souza et al. 1992, Castellani et al. 1995, Negrelle 1995, Dorneles \& Negrelle 1999, Falkenberg 1999, Citadini-Zanette et al. 2001, Rogalski \& Araújo 2005, Daniel 2006, Guimarães 2006, Negrelle 2006, Klein et al. 2007), considerando aproximadamente os $2.000 \mathrm{~km}^{2}$ de formações vegetais litorâneas distribuídas ao longo de $460 \mathrm{~km}$ de costa do Estado (Vibrans et al. 2012). No entanto, são raros os estudos que investigam o papel determinante das variáveis de solo sobre a estruturação das comunidades de restinga. Pesquisas com esse objetivo foram realizadas em restingas de outros Estados brasileiros, como Espírito Santo (Fabris 1995, Magnano et al. 2010), Paraná (Britez et al. 1997, Britez 2005), Pernambuco (Almeida Jr. et al. 2011), Piauí (Santos-Filho et al. 2013), Rio Grande do Sul (Costa et al. 2003) e São Paulo (Sztutman \&
Rodrigues 2002, Guedes et al. 2006) e identificaram diferenças estruturais nas comunidades vegetais ao longo da restinga em virtude das variações edáficas.

Considerando o exposto, este estudo teve como objetivo caracterizar a flora e as fitocomunidades de um gradiente de restinga no Estado de Santa Catarina, correlacionando-as com os atributos edáficos. Também avaliou-se a influência dos nutrientes e da disponibilidade hídrica do solo sobre a estrutura da vegetação ao longo do gradiente de restinga.

\section{Material e métodos}

O estudo foi realizado no Parque Estadual do Acaraí, Unidade de Conservação de proteção integral do município de São Francisco do Sul, litoral nordeste do Estado de Santa Catarina, Brasil, coordenadas $26^{\circ} 17^{\prime} \mathrm{S}$ e $48^{\circ} 33^{\prime} \mathrm{W}$. Compreende o maior remanescente de restinga em área contínua no Estado, sendo classificado como área de prioridade extremamente alta para a conservação da biodiversidade (PROBIO 2003). O clima, fortemente influenciado pela umidade marítima, é mesotérmico sem estação seca definida e com verões quentes ( $\mathrm{Cfa}$, segundo a classificação de Köppen), com índices pluviométricos médios de $1.874 \mathrm{~mm}$ ao ano (Knie 2002). Seu relevo caracteriza-se em planície costeira com 6.667 ha de área coberta por formação herbácea $(\mathrm{Rh})$, arbustiva (Ra), arbustivo-arbórea (Raa) e floresta de transição (Ft) (figura 1), além de outras formações menos representativas, como floresta submontana, várzeas e manguezais (FATMA2008). Os solos são classificados, segundo o Sistema Brasileiro de Classificação de Solos (EMBRAPA 2013), como: Neossolo Quartzarênico na formação herbácea, Espodossolo Ferrihumilúvico nas formações arbustiva e arbustivo-arbórea, e Espodossolo Ferrihumilúvico + Organossolo Háplico na floresta de transição (EPAGRI 2002). Tais classes de solo representam 90,3\% da área total do Parque, fracionadas em 15,1\% de Neossolo Quartzarênico de origem eólica e marinha e 75,2\% de Espodossolos de origem eólica. O tipo Espodossolo + Organossolo possui origem lagunar (Oliveira \& Vieira 2008).

Para o levantamento qualitativo da flora utilizou-se o wide patrolling (Filgueiras et al. 1994, Ratter et al. 2003). O critério de inclusão foi baseado na ocorrência de espermatófitas, excetuando-se epífitas e parasitas. O material botânico amostrado durante dois anos de coleta foi herborizado (Fidalgo \& Bononi 1989), identificado e incorporado ao Herbário Joinvillea da Universidade de Joinville (JOI). As 
identificações foram realizadas por comparações nos herbários Dr. Miguel Klein (FURB), Joinvillea (JOI), Museu Botânico Municipal de Curitiba (MBM), Universidade Federal do Paraná (UPCB) e por meio de consulta a especialistas. A lista de espécies seguiu as classificações de Judd et al. (2008) e APG III (2009), sendo também organizada conforme a ocorrência das espécies nas formações estudadas. Nomes de espécies e respectivos autores foram confirmados na Lista de Espécies da Flora do Brasil (2014).

Para a avaliação da estrutura das formações vegetais utilizou-se o método de parcelas (Felfili et al. 2011). Estas foram alocadas ao longo de três transectos perpendiculares à linha de costa e distantes $1,5 \mathrm{~km}$ entre si, sendo utilizadas cinco parcelas por formação em cada transecto, dispostas paralelas à costa. $\mathrm{Na}$ formação herbácea $(\mathrm{Rh})$ foram usadas parcelas de $1 \times 1 \mathrm{~m}$ (Munhoz \& Araújo 2011), distantes $1 \mathrm{~m}$ entre si. A cobertura das espécies foi estimada na escala de Causton (1988). Para cada espécie calculou-se a frequência e cobertura absoluta e relativa, e o valor de importância. Nas demais formações (Ra, Raa e Ft) foram utilizadas parcelas de $10 \times 10 \mathrm{~m}$ (Moro \& Martins 2011), distantes $10 \mathrm{~m}$ entre si. Na formação arbustiva, adotou-se como critério de inclusão o perímetro basal $\geq 5 \mathrm{~cm}$, enquanto que nas formações arbustivo-arbórea e florestal, adotou-se o perímetro a $1,30 \mathrm{~m}$ de altura do solo $\geq 10 \mathrm{~cm}$. Para essas formações foram avaliados a freqüência, densidade, dominância e o valor de importância (Mueller-Dombois \& Ellenberg 1974, Chapman 1976, Matteucci \& Colma 1982). Os índices de diversidade de Shannon $\left(H^{\prime}\right)$, com base no logaritmo natural, e de similaridade de Jaccard (Magurran 2013) foram calculados para cada uma das formações estudadas por meio do software Past (Hammer et al. 2001).
Para a caracterização nutricional dos solos de restinga, sessenta amostras de solo foram coletadas a uma profundidade de $15 \mathrm{~cm}$, sendo realizada uma coleta central por parcela ao longo das três transecções para as quatro formações, conforme EMBRAPA (2013). A cada cinco parcelas, as amostras foram misturadas de forma a se obter uma amostra composta, por transecto e por formação, totalizando 15 amostras. $\mathrm{A}$ análise química dos solos para $\mathrm{pH}$, fósforo $(\mathrm{P})$, potássio $(\mathrm{K})$, sódio $(\mathrm{Na})$, magnésio $(\mathrm{Mg})$, acidez potencial $\left(\mathrm{H}+\mathrm{Al}\right.$, íons $\mathrm{H}^{+}$e $\left.\mathrm{Al}^{3+}\right)$, soma de bases (SB), capacidade de troca catiônica (CTC), saturação por bases $(\mathrm{V})$ e matéria orgânica $(\mathrm{MO})$ foi realizada pelo Instituto Agronômico de Campinas (IAC). A disponibilidade hídrica do solo em cada formação foi estimada pela umidade gravimétrica tomada em 60 amostras de solo (EMBRAPA, 2013).

A comparação da similaridade da composição florística associada aos tipos de solo das formações de restinga estudadas foi realizada por análise de agrupamento no software Past (Hammer et al. 2001). Para explicar as diferenças estruturais entre as formações em função dos fatores edáficos foi empregado o teste de Mantel e adotado o protocolo bioenv (Clarke \& Ainsworth 1993). Para tanto, foram construídas duas matrizes, uma incluindo a abundânica das espécies e outra com as variáveis de solo. Para visualizar as respostas em relação às interações entre as espécies dentro das comunidades, as parcelas foram ordenadas em relação às variáveis de solo por uma análise de componentes principais (PCA); e a composição das comunidades, por se tratar de um gradiente em pequena escala espacial, por um escalonamento multidimensional não-métrico (NMDS) (Clarke \& Ainsworth 1993), sendo este método particularmente adequado para análise de

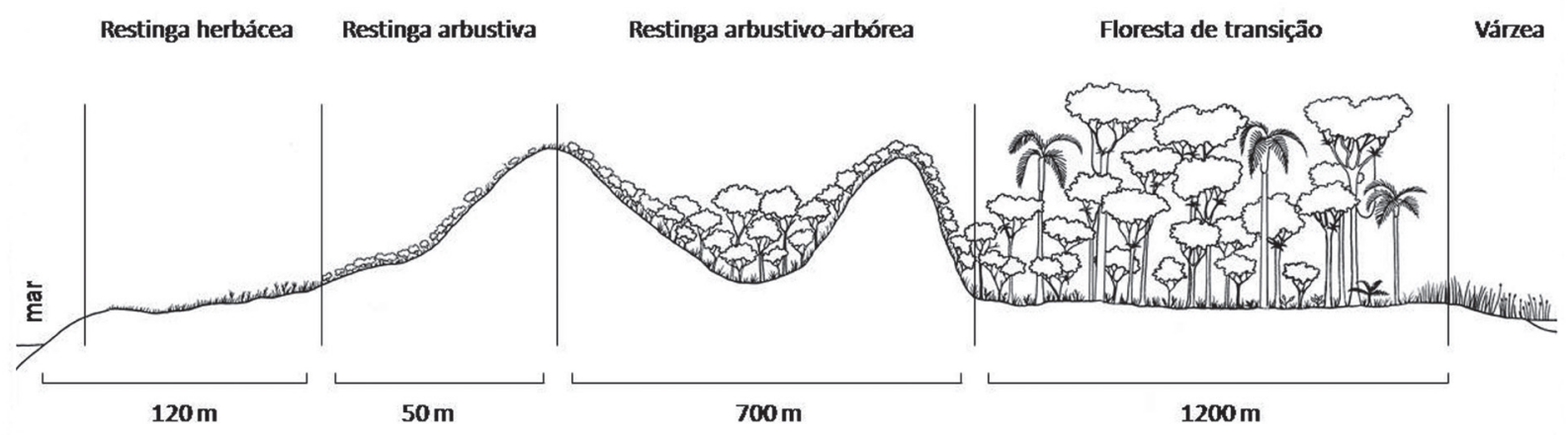

Figura 1. Formações da restinga do Parque Estadual do Acaraí, São Francisco do Sul, Estado de Santa Catarina, Brasil.

Figure 1. Restinga vegetation from the Parque Estadual do Acaraí, São Francisco do Sul, Santa Catarina State, Brazil. 
formações com elevada diversidade beta (De'ath 1999), como observado neste estudo. As espécies que mais caracterizaram as formações estudadas foram definidas pelo método do valor indicador (Dufrene \& Legendre 1997). Todas as análises multivariadas foram realizadas em ambiente $R$ versão 3.0 (Crawley 2007).

\section{Resultados e Discussão}

Diversidade Florística e Estrutura das Formações Nas quatro formações estudadas, 319 espécies pertencentes a 215 gêneros e 82 famílias foram identificadas (tabela 1, figura 2). As famílias mais representativas, em número de espécies, foram Asteraceae (35), Fabaceae (30), Myrtaceae (20), Rubiaceae (18) e Poaceae (13). Desse total, 244 espécies ocorreram de forma exclusiva nas formações vegetais, sendo 63 espécies exclusivas em Rh, 32 em Ra, 46 em Raa e 103 em Ft. As demais espécies foram compartilhadas entre uma ou mais formações. Maior coocorrência de espécies foi registrada entre Raa e Ft, com 42,6\% de similaridade. Apenas três espécies estiveram presentes em todas as fisionomias de restinga. A diversidade de espécies registrada em cada formação foi sumarizada na tabela 2, assim como as famílias de maior riqueza específica.

Uma nítida diferenciação florística entre as formações de restinga foi revelada pela baixa similaridade de Jaccard (figura 3). Entre Rh e Ra ocorreram apenas 11 espécies em comum, representando $7 \%$ das espécies amostradas nestas formações, com índice de similaridade de Jaccard de 0,100. Ra e Raa compartilharam 15 espécies, resultando num índice de similaridade de 0,207 , o que representa $8 \%$ das espécies amostradas nestas formações. Um total de 32 espécies ocorreu simultaneamente entre Raa e Ft. O índice de similaridade para estas duas formações foi de 0,230 , o que contribuiu com $12 \%$ do total de espécies nestas áreas. Os valores do índice de diversidade de Shannon mostraram que o ambiente florestal, sobre solo com alto teor de matéria orgânica (MO), é mais diverso $(3,29)$ quando comparado às demais formações (tabela 2). Ao contrário do esperado, o segundo maior índice $(2,89)$ foi observado em $\mathrm{Rh}$ sobre Neossolo Quartzarênico. O menor índice foi apresentado pela formação de Ra $(2,47)$, desenvolvida sobre Espodossolo.

As espécies de maior importância na estrutura das comunidades, apesar de apresentarem ranqueamento distinto entre os valores de importância obtidos no levantamento fitossociológico e nos resultados gerados pelo método do valor indicador (indval), foram, na sua maioria, as mesmas (tabelas 3 e 4). Desta forma, as cinco espécies mais características de cada fisionomia foram: Smilax campestris, Scaevola plumieri, Canavalia rosea, Stylosanthes viscosa e Blutaparon portulacoides em Rh; Guapira opposita, Myrcia pulchra, Norantea brasiliensis, Clusia criuva e Psidium cattleianum em Ra; Ocotea pulchella, Pera glabrata, Ternstroemia brasiliensis, Myrsine venosa e Ilex theezans em Raa; e Calophyllum brasiliense, Alchornea triplinervia, Nectandra oppositifolia, Schinus terebinthifolius e Andira fraxinifolia em Ft.

A riqueza de espécies registrada neste estudo, exibida pelo índice de diversidade de Shannon, mostrou-se expressiva quando comparada a outras áreas de restinga estudadas no Estado, principalmente para as fisionomias da restinga herbácea (Cordazzo \& Costa 1989, Souza et al. 1992, Castellani et al. 1995, Falkenberg 1999, Daniel 2006, Guimarães 2006, Klein et al. 2007) e da restinga arbustiva (Danilevicz et al. 1990, Korte et al. 2013). Os componentes arbustivoarbóreo e florestal apresentaram riqueza similar quando comparados ao levantamento realizado em área de planície quaternária do município de Volta Velha, SC (Negrelle 2006). De maneira geral, a riqueza de espécies aumentou em direção à formação florestal; mas em Ra, a baixa diversidade pode decorrer da pequena extensão territorial ocupada por esta fisionomia, além dos fatores edáficos restritivos. As famílias com maior número de espécies (Asteraceae, Fabaceae, Myrtaceae, Rubiaceae e Poaceae) são típicas de outras áreas de restinga dos Estados do sul do Brasil (Silva 1990, Santos et al. 2012, Korte et al. 2013). Entre estas famílias, Myrtaceae e Fabaceae têm elevada riqueza de espécies em áreas de restinga localizadas nas regiões sudeste, nordeste e norte do país (Oliveira-Filho \& Carvalho 1993, Pereira et al. 2001, Assis et al. 2004, Guedes et al. 2006, Almeida Jr. \& Zickel 2009, Sá \& Araujo 2009, Dantas et al. 2010, Silva et al. 2010, Almeida Jr. et al. 2011, Giareta et al. 2013). Estas famílias são reconhecidas como umas das principais famílias neotropicais (Gentry 1988), com grande representatividade na vertente costeira da Mata Atlântica (Salimon \& Negrelle 2001) e que melhor caracterizam a flora das restingas do Brasil (Klein 1982, Araujo \& Henriques 1984). Assim, a área de estudo tem uma das áreas contínuas de restinga mais bem preservadas do Estado, com vegetação distribuída em distintas formações e estrutura de comunidades bem demarcadas pelo gradiente edáfico. 
Tabela 1. Espécies amostradas nas formações da restinga do Parque Estadual do Acaraí, São Francisco do Sul, Estado de Santa Catarina, Brasil. No Col.: Número de coleta de João Carlos Ferreira de Melo Júnior. Formas de vida (Fv): erva (e), subarbusto (sa), arbusto (ab), arvoreta (at), árvore (av) e liana (1). Formações Vegetais: restinga herbácea (Rh), restinga arbustiva $(\mathrm{Ra})$, restinga arbustivo-arbórea (Raa) e floresta de transição (Ft).

Table 1. Species sampled in the restinga vegetation of the Parque Estadual do Acaraí, São Francisco do Sul, Santa Catarina State, Brazil. No Col.: Collector number of João Carlos Ferreira de Melo Júnior. Life forms (Fv): herb (e), subshrub (sa), shrub (ab), small tree (at), tree (av), and liana (1). Vegetation: Herb restinga (Rh), Shrub restinga (Ra), Shrub-tree restinga (Raa), and Transition forest (Ft).

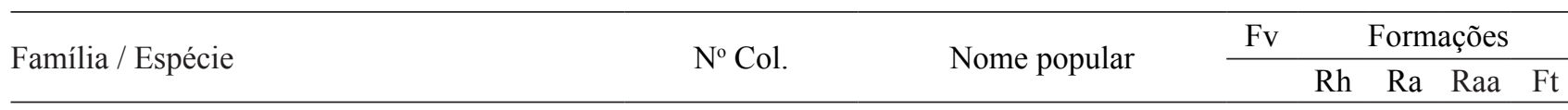

Acanthaceae

Justicia brasiliana Roth

1070

Aizoaceae

Sesuvium portulacastrum (L.) L.

Amaranthaceae

Alternanthera littoralis P.Beauv.

Amaranthus lividus L.

Blutaparon portulacoides (A.St.-Hil.) Mears

Chenopodium retusum (Moq.) Moq.

Chenopodium sp.

Amarylidaceae

Bomarea edulis (Tussac) Herb.

Anacardiaceae

Schinus terebinthifolius Raddi

Tapirira guianensis Aubl.

1034

1208

1271

1004

1149

1002

1272

1055

1273

Annonaceae

Annona glabra L.

1274

Annona neosericea H.Rainer

1184

Annona sylvatica A.St.-Hil.

1275

Duguetia lanceolata A.St.Hill.

1276

Guatteria australis A.St.-Hil.

Apiaceae

Centella asiatica (L.) Urb.

Cyclospermum leptophyllum (Pers.) Britton P.Wilson

1156

1041

1209

Apocynaceae

Gonioanthela hilariana (E. Fourn.) Malme

Oxypetalum tomentosum Wight ex Hook. \& Arn.

1277

1008

Prestonia coalita (Vell.) Woodson

1210

Tabernaemontana catharinensis A. DC.

1278

Temnadenia stellaris (Lindl.) Miers

1279

Aquifoliaceae

Ilex brevicuspis Reissek

Ilex dumosa Reissek

$\begin{array}{ll}\text { Annona neosericea H.Rainer } & 1184 \\ \text { Annona sylvatica A.St.-Hil. } & 1275 \\ \text { Duguetia lanceolata A.St.Hill. } & 1276 \\ \text { Guatteria australis A.St.-Hil. } & 1156\end{array}$

1238

justicia-vermelha

$\mathrm{ab}$

bredo-da-praia

e X

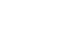

277

$\begin{array}{cccc} & \text { e } & \text { X } & \\ \text { caruru-de-folha-cuia } & \text { e } & & \text { X } \\ \text { capotiraguá } & \text { e } & \text { X } \\ \text { pega-pinto } & \text { e } & \text { X } \\ & \text { e } & \text { X }\end{array}$

$\begin{array}{cllll}\text { aroeira-vermelha } & \text { av } & X & X & X \\ \text { pau-pombo } & \text { av } & & & X\end{array}$

cortiça-do-brejo av

araticum av

araticum av

pindavuna

embiú

centelha

aipo-bravo

cipó-de-leite

leiteiro-de-vaca

cipó-de-leite

caúna-da-serra

erva-piriquita e

X

e X

e $\mathrm{X}$

1

X

e X

1

av

X

X

$\mathrm{X}$

$\mathrm{X}$

$\mathrm{X}$

$\mathrm{X}$

$\mathrm{X}$

X X

1

X

$\mathrm{X}$

at $\mathrm{X} \quad \mathrm{X}$

at $\mathrm{X}$ 
Tabela 1 (continuação)

Família / Espécie $\quad \mathrm{N}^{\circ} \mathrm{Col}$

Ilex pseudobuxus Reissek

Ilex theezans Mart. ex Reissek

Araceae

Anthurium sp.

Araliaceae

Hydrocotyle bonariensis Lam.

Oreopanax capitatus (Jacq.) Decne. \& Planch.

Arecaceae

Attalea dubia (Mart.) Burret

Bactris setosa Mart.

Butia catarinensis Noblick \& Lorenzi

Euterpe edulis Mart.

Geonoma elegans Mart.

Geonoma schottiana Mart.

Syagrus romanzoffiana (Cham.) Glassman

Aristolochiaceae

Aristolochia trilobata $\mathrm{L}$.

Asteraceae

Achyrocline alata DC.

Achyrocline satureioides (Lam.) DC.

Ageratum conyzoides L.

Austroeupatorium inulaefolim (Kunth) R.M.

King \& H. Rob.

Austroeupatorium picturatum (Malme)

R.M.King \& H. Rob.

Baccharis crispa Spreng.

Baccharis dracunculifolia DC.

Baccharis illinita DC.

Baccharis junciformis DC.

Baccharis microdonta DC.

Baccharis singularis (Vell.) G.M. Barroso

Baccharis sphenophylla Dusén ex Malme

Baccharis trimera (Less.) DC.

Bidens alba (L.) DC.

Conyza primulifolia (Lam.) Cuatrec. \&

Lourteig

Cyrtocymura scorpioides (Lam.) H.Rob.

Emilia fosbergii Nicolson

Eupatorium laevigatum Lam.

Eupatorium littorale Cabrera

Gamochaeta americana (Mill.) Wedd.

Hieracium commersonii Monnier

1116

1073

1280

1011

1211

1281

1196

1198

1282

1239

1202

1197

1350

1225

1212

1213

1351

1111

1352

1035

1219

1220

1082

1063

1058

1283

1087

1353

1014

1191

1139

1221

1006

1214
Nome popular

caúna-branca

caúna-preta

antúrio

erva-capitão

indaiá

tucum

butiá

palmito-juçara

ouricanga

aricanga

jerivá

milhomem

marcela

marcela-do-campo

mentrasto

erva-de-embira

erva-de-embira

carqueja

alecrim-do-campo

carqueja

vassoura

vassoura-branca

vassoura

vassoura

carqueja

picão

buva

erva-preá

pincel

cambará-falso

eupatório

macelinha

\begin{tabular}{cccc} 
Fv & \multicolumn{3}{c}{ Formações } \\
\hline $\mathrm{Rh}$ & $\mathrm{Ra}$ & $\mathrm{Raa}$ & $\mathrm{Ft}$
\end{tabular}

at

$\mathrm{ab}$

X $\quad X \quad X$

e

$\mathrm{X}$

e $\mathrm{X}$

av $\mathrm{X}$

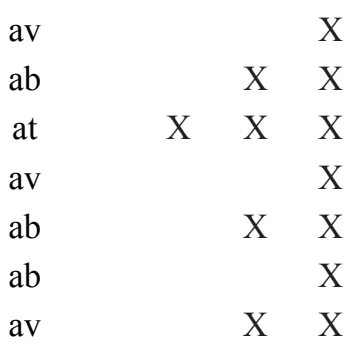

$1 \quad \mathrm{X}$

e $\mathrm{X} \quad \mathrm{X}$

e $X$

e $\mathrm{X}$

sa $\quad X$

ab $\quad \mathrm{X}$

sa $\mathrm{X}$

$\begin{array}{lll}\mathrm{ab} & \mathrm{X} & \mathrm{X}\end{array}$

sa $\mathrm{X}$

ab $\quad X$

ab $\quad X$

ab $\quad X \quad X$

ab $\quad X$

sa $X$

e $\mathrm{X}$

sa $\mathrm{X}$

sa $\mathrm{X} \quad \mathrm{X}$

e $\quad X$

ab $\quad X \quad X$

ab $\quad X$

e $\mathrm{X}$

e X 
Tabela 1 (continuação)

\begin{tabular}{|c|c|c|c|c|c|c|c|}
\hline \multirow{2}{*}{ Família / Espécie } & \multirow{2}{*}{ № Col. } & \multirow{2}{*}{ Nome popular } & \multirow[t]{2}{*}{$\mathrm{Fv}$} & \multicolumn{4}{|c|}{ Formações } \\
\hline & & & & $\mathrm{Rh}$ & $\mathrm{Ra}$ & Raa & $\mathrm{Ft}$ \\
\hline Mikania cordifolia (L. f.) Willd. & 1240 & guaco & 1 & & & $\mathrm{X}$ & \\
\hline Mikania involucrata Hook. \& Arn. & 1030 & guaco & 1 & & & $\mathrm{X}$ & \\
\hline Mikania laevigata Sch. Bip. ex Baker & 1241 & guaco & 1 & & & $\mathrm{X}$ & \\
\hline Mikania paniculata DC. & 1242 & guaco & 1 & & & $\mathrm{X}$ & \\
\hline Mikania trinervis Hook. \& Arn. & 1243 & & 1 & & & $\mathrm{X}$ & \\
\hline Noticastrum calvatum (Baker) Cuatrec. & 1353 & & sa & $\mathrm{X}$ & & & \\
\hline Pterocaulon lorentzii Malme & 1033 & & sa & $\mathrm{X}$ & & & \\
\hline Pterocaulon polypterum (DC.) Cabrera & 1046 & & sa & & $\mathrm{X}$ & & \\
\hline Senecio crassiflorus (Poir.) DC. & 1037 & margarida-da-praia & $\mathrm{e}$ & $\mathrm{X}$ & & & \\
\hline Solidago chilensis Meyen & 1124 & arnica & $\mathrm{e}$ & & $\mathrm{X}$ & & \\
\hline Sphagneticola trilobata (L.) Pruski & 1222 & vedélia & $\mathrm{e}$ & & $\mathrm{X}$ & & \\
\hline Symphyopappus casarettoi B.L. Rob. & 1016 & vassoura-do-campo & $\mathrm{ab}$ & $\mathrm{X}$ & $\mathrm{X}$ & $\mathrm{X}$ & $\mathrm{X}$ \\
\hline $\begin{array}{l}\text { Symphyopappus reitzii (Cabrera) R.M.King } \\
\text { \& H.Rob. }\end{array}$ & 1223 & vassoura & $\mathrm{ab}$ & & $\mathrm{X}$ & $\mathrm{X}$ & \\
\hline Vernonanthura puberula (Less.) H. Rob. & 1203 & vassoura & $\mathrm{ab}$ & & $\mathrm{X}$ & & \\
\hline \multicolumn{8}{|l|}{ Begoniaceae } \\
\hline Begonia cucullata Willd. & 1287 & begônia-do-brejo & e & & & & $\mathrm{X}$ \\
\hline \multicolumn{8}{|l|}{ Bignoniaceae } \\
\hline Dolichandra unguis-cati (L.) L.G.Lohmann & 1285 & cipó-de-gato & 1 & & & & $\mathrm{X}$ \\
\hline Handroanthus umbellatus (Sond.) Mattos & 1286 & ipê-amarelo & av & & & & $\mathrm{X}$ \\
\hline Jacaranda puberula Cham. & 1354 & caroba & av & & & & $\mathrm{X}$ \\
\hline \multicolumn{8}{|l|}{ Bixaceae } \\
\hline Bixa orellana $\mathrm{L}$. & 1284 & urucum & av & & & & $\mathrm{X}$ \\
\hline \multicolumn{8}{|l|}{ Boraginaceae } \\
\hline Cordia sellowiana Cham. & 1291 & louro-mole & av & & & & $\mathrm{X}$ \\
\hline Varronia curassavica (Jacq.) Roem. \& Schult. & 1013 & erva-baleeira & $\mathrm{ab}$ & $\mathrm{X}$ & $\mathrm{X}$ & $\mathrm{X}$ & $\mathrm{X}$ \\
\hline \multicolumn{8}{|l|}{ Bromeliaceae } \\
\hline Aechmea gamossepala Wittm. & 1100 & bromélia & e & & $\mathrm{X}$ & $\mathrm{X}$ & \\
\hline Aechmea ornata Baker & 1224 & bromélia & $\mathrm{e}$ & & $\mathrm{X}$ & & \\
\hline Ananas fritzmuelleri Camargo & 1225 & ananás & $\mathrm{e}$ & & $\mathrm{X}$ & $\mathrm{X}$ & $\mathrm{X}$ \\
\hline Bromelia antiacantha Bertol. & 1226 & brimélia & $\mathrm{e}$ & & $\mathrm{X}$ & $\mathrm{X}$ & \\
\hline Dyckia maritima Baker & 1215 & gravatá & $\mathrm{e}$ & $\mathrm{X}$ & $\mathrm{X}$ & & \\
\hline Neoregelia laevis (Mez) L.B.Sm. & 1244 & bromélia & $\mathrm{e}$ & & & $\mathrm{X}$ & \\
\hline Nidularium innocentii Lem. & 1245 & bromélia & $\mathrm{e}$ & & & $\mathrm{X}$ & \\
\hline Nidularium procerum Lindm. & 1246 & bromélia & e & & & $\mathrm{X}$ & \\
\hline \multicolumn{8}{|l|}{ Cactaceae } \\
\hline Nopalea cochenillifera (L.) salm-Dyck & 1227 & palma & e & & $\mathrm{X}$ & & \\
\hline Opuntia arechavaletai Speg. & 1228 & palma & $\mathrm{ab}$ & & $\mathrm{X}$ & $\mathrm{X}$ & \\
\hline Opuntia monacantha Haw. & 1192 & palma & $\mathrm{ab}$ & $\mathrm{X}$ & $\mathrm{X}$ & & \\
\hline \multicolumn{8}{|l|}{ Calophyllaceae } \\
\hline Calophyllum brasiliense Camb. & 1072 & olandi & av & & & $\mathrm{X}$ & $\mathrm{X}$ \\
\hline \multicolumn{8}{|l|}{ Calyceraceae } \\
\hline Acicarpha bonariensis (Pers.) Herter & 1012 & roseta-de-espinho & $\mathrm{e}$ & $\mathrm{X}$ & & & \\
\hline
\end{tabular}


Tabela 1 (continuação)

Família / Espécie

Cannaceae

Canna indica L.

Capparaceae

Cynophalla flexuosa (L.) J.Presl

Caryophyllaceae

Cardionema ramosissima (Weinm.) A.Nelson \& J. F. Macbr.

Celastraceae

Maytenus robusta Reissek

Maytenus gonoclada Mart.

1128

Chrysobalanaceae

Hirtella hebeclada Moric. ex DC.

Clethraceae

Clethra scabra Pers.

Clusiaceae

Clusia criuva Cambess.

1078

Garcinia gardneriana (Planch. \& Triana) Zappi

1247

Commelinaceae

Commelina erecta $\mathrm{L}$.

1093

Commelina sp.

1248

Convolvulaceae

Ipomoea imperati (Vahl) Griseb.

Ipomoea pes-caprae (L.) R. Br.

1003

1032

Cyperaceae

Cyperus aggregatus (Willd.) Endl.

1029

Cyperus chalaranthus J. Presl \& C.Presl

1151

Cyperus ligularis L.

1052

Cyperus luzulae (L.) Retz.

1150

Eleocharis geniculata Roem. \& Schult.

Fimbristylis cymosa R. Br.

1130

1020

Remirea maritima Aubl.

1015

Rhynchospora brasiliensis Boeckeler

1129

Scleria hirtella Sw.

1295

Dilleniaceae

Davilla rugosa Poir.

Tetracera sellowiana Schltdl.

Dioscoreaceae

Dioscorea laxiflora Mart. ex Griseb.

Elaeocarpaceae

Sloanea guianensis (Aubl.) Benth.

Sloanea lasiocoma K. Schum.
1205

1292
Nome popular

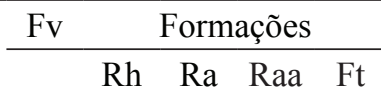

e

X

feijão-bravo

$\mathrm{ab}$

X

roseta

e X

cafezinho-do-mato

cafezinho-do-mato

$\mathrm{ab}$

X

at

X X

cinzeiro

av

X

$$
\text { carne-de-vaca }
$$

$\mathrm{ab}$

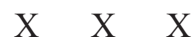

mangue-de-formiga bacopari

$\mathrm{ab}$

av

$\begin{array}{lll}X & X & X\end{array}$

X X

trapoeraba

trapoerada

$\begin{array}{lll}\text { e } & X & \\ \text { e } & & X\end{array}$

cipó-da-praia

pé-de-cabra

e $X$

e X

tiririca

tiririca

tiririca

tiririca

capim

pinheirinho-da-praia

junco-de-cobra

cipó-caboclo

cipó-vermelho

cará

e X

e $X$

e X

e $\mathrm{X}$

e

X

e $X$

e X

e $X$

e

X

X

X

X

laranjeira-do-mato sapopema at

av
X

X 
Tabela 1 (continuação)

\begin{tabular}{|c|c|c|c|c|c|c|c|}
\hline \multirow{2}{*}{ Família / Espécie } & \multirow{2}{*}{$\mathrm{N}^{\circ}$ Col. } & \multirow{2}{*}{ Nome popular } & \multirow[t]{2}{*}{$\mathrm{Fv}$} & \multicolumn{4}{|c|}{ Formações } \\
\hline & & & & $\mathrm{Rh}$ & $\mathrm{Ra}$ & Raa & $\mathrm{Ft}$ \\
\hline \multicolumn{8}{|l|}{ Ericaceae } \\
\hline Gaylussacia brasiliensis (Spreng.) Meisn. & 1230 & camarinha & $a b$ & & $\mathrm{X}$ & $\mathrm{X}$ & \\
\hline \multicolumn{8}{|l|}{ Erythroxylaceae } \\
\hline Erythroxylum amplifolium (Mart.) O. E. Schulz & 1083 & cocão & at & & $\mathrm{X}$ & $\mathrm{X}$ & \\
\hline Erythroxylum cuspidifolium Mart & 1146 & caga-fede & $a b$ & & $\mathrm{X}$ & & \\
\hline Erythroxylum deciduum A. St.-Hil. & 1250 & cocão & at & & & $\mathrm{X}$ & \\
\hline \multicolumn{8}{|l|}{ Euphorbiaceae } \\
\hline Alchornea glandulosa Poepp. \& Endl. & 1293 & tanheiro & av & & & & $\mathrm{X}$ \\
\hline Alchornea triplinervia (Spreng.) Müll. Arg. & 1164 & tanheiro & av & & & $\mathrm{X}$ & $\mathrm{X}$ \\
\hline Euphorbia cyathophora Murray & 1187 & & $\mathrm{e}$ & & & & $\mathrm{X}$ \\
\hline Euphorbia hyssopifolia $\mathrm{L}$. & 1087 & erva-de-andorinha & e & $\mathrm{X}$ & & & \\
\hline Gymnanthes klotzschiana Müll.Arg. & 1251 & branquilho & at & & & $\mathrm{X}$ & \\
\hline Microstachys corniculata (Vahl) Griseb. & 1091 & granxuma-de-chifre & $\mathrm{e}$ & $\mathrm{X}$ & & & \\
\hline Sapium glandulatum (Vell.) Pax & 1294 & pau-leiteiro & av & & & & $\mathrm{X}$ \\
\hline \multicolumn{8}{|l|}{ Fabaceae } \\
\hline $\begin{array}{l}\text { Abarema langsdorffii (Benth.) Barneby \& J. W. } \\
\text { Grimes }\end{array}$ & 1077 & olho-de-cabra & av & & & $\mathrm{X}$ & \\
\hline Andira anthelmia (Vell.) Benth. & 1079 & árvore-de-morcego & av & & & $\mathrm{X}$ & \\
\hline Andira fraxinifolia Benth. & 1126 & árvore-doce & av & & & $\mathrm{X}$ & $\mathrm{X}$ \\
\hline $\begin{array}{l}\text { Balizia pedicelaris (DC.) Barneby \& J. W. } \\
\text { Grimes }\end{array}$ & 1109 & timboúva & av & & & $\mathrm{X}$ & \\
\hline Canavalia rosea (Sw.) DC. & 1000 & feijão-da-praia & e & $\mathrm{X}$ & & & \\
\hline Centrosema virginianum (L.) Benth. & 1007 & feijão-bravo & 1 & $\mathrm{X}$ & & & \\
\hline Chamaecrista flexuosa (L.) Greene & 1026 & peninha & $\mathrm{e}$ & $\mathrm{X}$ & & & \\
\hline Clitoria falcata Lam. & 1106 & feijão-do-mato & as & & $\mathrm{X}$ & & \\
\hline Dalbergia ecastaphyllum (L.) Taub. & 1048 & marmeleiro-da-praia & as & & $\mathrm{X}$ & & \\
\hline Dalbergia frutescens (Vell.) Britton & 1048 & & av & & & & $\mathrm{X}$ \\
\hline Desmodium adscendens (Sw.) DC. & 1028 & pega-pega & $\mathrm{e}$ & $\mathrm{X}$ & $\mathrm{X}$ & & \\
\hline Desmodium barbatum (L.) Benth. & 1047 & pega-pega & e & $\mathrm{X}$ & & & \\
\hline Desmodium incanum DC. & 1040 & carrapicho-beiço-de-boi & e & $\mathrm{X}$ & $\mathrm{X}$ & & \\
\hline Dioclea wilsonii Standl. & 1195 & olho-de-boi & 1 & & & & $\mathrm{X}$ \\
\hline Erythrina falcata Benth. & 1296 & corticeira & av & & & & $\mathrm{X}$ \\
\hline Erythrina speciosa Andrews & 1301 & mulungu & av & & & & $\mathrm{X}$ \\
\hline Indigofera suffruticosa Mill. & 1231 & anileiro & $a b$ & & $\mathrm{X}$ & & \\
\hline Inga marginata Willd. & 1200 & ingá-mirim & av & & & & $\mathrm{X}$ \\
\hline Inga sessilis (Vell.) Mart. & 1118 & ingá & av & & & $\mathrm{X}$ & $\mathrm{X}$ \\
\hline Lonchocarpus sericeus (Poir.) Kunth ex DC. & 1140 & embira-branca & $a b$ & & $\mathrm{X}$ & & \\
\hline Mimosa bimucronata (DC.) Kuntze & 1298 & silva & $a b$ & & & & $\mathrm{X}$ \\
\hline Ormosia arborea Harms & 1252 & olho-de-cabra & av & & & $\mathrm{X}$ & \\
\hline Schizolobium parahyba (Vell.) Blake & 1304 & guarapuvu & av & & & & $\mathrm{X}$ \\
\hline Senna bicapsularis (L.) Roxb. & 1110 & fedegoso & av & & & $\mathrm{X}$ & \\
\hline $\begin{array}{l}\text { Senna macranthera (DC. ex Collad.) H. S. } \\
\text { Irwin \& Barneby }\end{array}$ & 1299 & pau-fava & av & & & & $\mathrm{X}$ \\
\hline
\end{tabular}


Tabela 1 (continuação)

\begin{tabular}{|c|c|c|c|c|c|c|c|}
\hline \multirow{2}{*}{ Família / Espécie } & \multirow{2}{*}{$\mathrm{N}^{\circ}$ Col. } & \multirow{2}{*}{ Nome popular } & \multirow[t]{2}{*}{$\mathrm{Fv}$} & \multicolumn{4}{|c|}{ Formações } \\
\hline & & & & $\mathrm{Rh}$ & $\mathrm{Ra}$ & Raa & $\mathrm{Ft}$ \\
\hline $\begin{array}{l}\text { Senna pendula (Humb.\& Bonpl. ex Willd.) H. } \\
\text { S. Irwin \& Barneby }\end{array}$ & 1142 & aleluia & $\mathrm{ab}$ & & $\mathrm{X}$ & & \\
\hline Sophora tomentosa $\mathrm{L}$. & 1121 & feijão-da-praia & at & & $\mathrm{X}$ & $\mathrm{X}$ & \\
\hline Stylosanthes viscosa (L.) Sw. & 1021 & vissitudo & as & $\mathrm{X}$ & & & \\
\hline Zollernia ilicifolia (Brongn.) Vogel & 1172 & falsa-espinheira-santa & av & & & & $\mathrm{X}$ \\
\hline Zornia latifolia $\mathrm{Sm}$. & 1188 & zórnia & sa & & $\mathrm{X}$ & $\mathrm{X}$ & \\
\hline \multicolumn{8}{|l|}{ Goodeniaceae } \\
\hline Scaevola plumieri (L.) Vahl & 1101 & mangue-da-praia & sa & $\mathrm{X}$ & & & \\
\hline \multicolumn{8}{|l|}{ Iridaceae } \\
\hline Sisyrinchium vaginatum Spreng. & 1314 & canchalágua & $\mathrm{e}$ & & & & $\mathrm{X}$ \\
\hline \multicolumn{8}{|l|}{ Juncaceae } \\
\hline Juncus marginatus Rostk. & 1317 & junco & $\mathrm{e}$ & & & & $\mathrm{X}$ \\
\hline Juncus sp. & 1302 & junco & e & $\mathrm{X}$ & & & \\
\hline \multicolumn{8}{|l|}{ Lauraceae } \\
\hline Aiouea saligna Meisn. & 1253 & canela-anhuíba & av & & & $\mathrm{X}$ & \\
\hline Endlicheria paniculata (Spreng.) J.F.Macbr. & 1355 & canela-branca & av & & & & $\mathrm{X}$ \\
\hline Nectandra oppositifolia Nees \& Mart. ex Nees & 1101 & canela-garuva & av & & & $\mathrm{X}$ & $\mathrm{X}$ \\
\hline Ocotea aciphylla (Nees \& Mart.) Mez & 1356 & louro-fofo & av & & & & $\mathrm{X}$ \\
\hline Ocotea pulchella (Nees \& Mart.) Mez & 1085 & canela-do-brejo & $\mathrm{ab}$ & & $\mathrm{X}$ & $\mathrm{X}$ & $\mathrm{X}$ \\
\hline \multicolumn{8}{|l|}{ Loganiaceae } \\
\hline Strychnos trinervis (Vell.) Mart. & 1357 & cipó-cruzeiro & 1 & & & $\mathrm{X}$ & \\
\hline \multicolumn{8}{|l|}{ Malpighiaceae } \\
\hline Bunchosia pallescens Skottsb. & 1185 & & av & & & & $\mathrm{X}$ \\
\hline Byrsonima ligustrifolia A. Juss. & 1306 & baga-de-tucano & av & & & & $\mathrm{X}$ \\
\hline Heteropterys aenea Griseb & 1167 & & 1 & & $\mathrm{X}$ & $\mathrm{X}$ & \\
\hline Heteropterys nitida (Lam.) DC. & 1312 & & 1 & & & & $\mathrm{X}$ \\
\hline Stigmaphyllon affine A. Juss. & 1096 & & $\mathrm{e}$ & & $\mathrm{X}$ & & \\
\hline Tetrapterys phlomoides (Spreng.) Nied. & 1311 & & 1 & & & & $\mathrm{X}$ \\
\hline \multicolumn{8}{|l|}{ Malvaceae } \\
\hline Hibiscus tiliaceus L. & 1194 & algodoeiro-da-praia & $a b$ & & $\mathrm{X}$ & & \\
\hline Pavonia sp.1 & 1102 & & sa & $\mathrm{X}$ & & & \\
\hline Pavonia sp.2 & 1122 & & sa & & $\mathrm{X}$ & & \\
\hline Pseudobombax grandiflorum (Cav.) A. Robyns & 1358 & embiruçu & av & & & & $\mathrm{X}$ \\
\hline Sida carpinifolia $\mathrm{L}$ & 1068 & mata-pasto & sa & $\mathrm{X}$ & $\mathrm{X}$ & & \\
\hline Waltheria indica $\mathrm{L}$. & 1307 & douradinha & $\mathrm{e}$ & $\mathrm{X}$ & & & \\
\hline \multicolumn{8}{|l|}{ Marantaceae } \\
\hline Calathea monophylla (Vell.) Körn. & 1206 & caeté & e & & & $X$ & $\mathrm{X}$ \\
\hline Stromanthe tonckat (Aubl.) Eichler & 1303 & pariquera-açu & e & & & & $\mathrm{X}$ \\
\hline \multicolumn{8}{|l|}{ Marcgraviaceae } \\
\hline Norantea brasiliensis Choisy & 1087 & cachimbeira & $a b$ & & $\mathrm{X}$ & & \\
\hline \multicolumn{8}{|l|}{ Melastomataceae } \\
\hline Clidemia hirta (L.) D.Don & 1232 & pixirica & $\mathrm{ab}$ & & $\mathrm{X}$ & & \\
\hline Leandra fragilis Cogn. & 1233 & & $\mathrm{sa}$ & & $\mathrm{X}$ & & \\
\hline
\end{tabular}


Tabela 1 (continuação)

\begin{tabular}{|c|c|c|c|c|c|c|c|}
\hline \multirow{2}{*}{ Família / Espécie } & \multirow{2}{*}{$\mathrm{N}^{\circ}$ Col. } & \multirow{2}{*}{ Nome popular } & \multirow[t]{2}{*}{$\mathrm{Fv}$} & \multicolumn{4}{|c|}{ Formações } \\
\hline & & & & $\mathrm{Rh}$ & $\mathrm{Ra}$ & Raa & $\mathrm{Ft}$ \\
\hline Miconia cubatanensis Hoehne & 1253 & pixirica & av & & & $\mathrm{X}$ & \\
\hline Miconia pusilliflora (DC.) Naudin & 1071 & pixirica & at & & & $\mathrm{X}$ & $\mathrm{X}$ \\
\hline Tibouchina clavata (Pers.) Wurdack & 1027 & orelha-de-onça & $\mathrm{sa}$ & $\mathrm{X}$ & $\mathrm{X}$ & & \\
\hline Tibouchina pulchra Cogn. & 1305 & jacatirão & av & & & & $\mathrm{X}$ \\
\hline Tibouchina tricopoda (DC.) Baill. & 1254 & quaresmeira & av & & & $\mathrm{X}$ & \\
\hline Tibouchina sp. & 1359 & & av & & & $\mathrm{X}$ & $\mathrm{X}$ \\
\hline \multicolumn{8}{|l|}{ Meliaceae } \\
\hline Cedrela fissilis Vell. & 1207 & cedro & av & & & & $\mathrm{X}$ \\
\hline Guarea macrophylla Vahl & 1255 & bago-de-cachorro & av & & & $\mathrm{X}$ & $\mathrm{X}$ \\
\hline Trichilia casaretti C. DC. & 1360 & catiguá-vermelho & av & & & & $\mathrm{X}$ \\
\hline Trichilia lepidota Mart. & 1256 & catiguá-morcego & av & & & $\mathrm{X}$ & \\
\hline \multicolumn{8}{|l|}{ Menyanthaceae } \\
\hline Nymphoides indica (L.) Kuntze & 1095 & ninfeia & $\mathrm{e}$ & $\mathrm{X}$ & & & \\
\hline \multicolumn{8}{|l|}{ Molluginaceae } \\
\hline Mollugo verticillata $\mathrm{L}$. & 1103 & capim-tapete & $\mathrm{e}$ & $\mathrm{X}$ & & & \\
\hline \multicolumn{8}{|l|}{ Monimiaceae } \\
\hline Mollinedia schottiana (Spreng.) Perkins & 1257 & erva-santa & at & & & $\mathrm{X}$ & $\mathrm{X}$ \\
\hline Mollinedia fruticulosa Perkins & 1319 & erva-santa & at & & & $\mathrm{X}$ & \\
\hline \multicolumn{8}{|l|}{ Moraceae } \\
\hline Ficus adhatodifolia Schott ex Spreng. & 1258 & gameleira & av & & & & $\mathrm{X}$ \\
\hline Ficus citrifolia Mill. & 1320 & figueira & av & & & & $\mathrm{X}$ \\
\hline Ficus enormis Mart. ex Miq. & 1336 & figueira & av & & & & $\mathrm{X}$ \\
\hline Ficus insipida Willd. & 1065 & figueira-branca & av & & & $\mathrm{X}$ & $\mathrm{X}$ \\
\hline Ficus luschnathiana Miq. & 1108 & figueira-do-mato & av & & & $\mathrm{X}$ & $\mathrm{X}$ \\
\hline Ficus organensis (Miq.) Miq. & 1327 & figueira-da-folha-miúda & av & & & & $\mathrm{X}$ \\
\hline Ficus pertusa L. f. & 1259 & mata-pau & av & & & $\mathrm{X}$ & $\mathrm{X}$ \\
\hline Ficus trigona $\mathrm{L} . \mathrm{f}$. & 1260 & gameleira & av & & & $\mathrm{X}$ & \\
\hline Maclura tinctoria Gaudich. & 1335 & taiuva & av & & & & $\mathrm{X}$ \\
\hline Sorocea bonplandii (Baill.) W.C.Burger et al. & 1361 & falsa-espinheira-santa & av & & & $\mathrm{X}$ & $\mathrm{X}$ \\
\hline \multicolumn{8}{|l|}{ Myrtaceae } \\
\hline Calyptranthes strigipes O.Berg & 1308 & araçazinho & $\mathrm{ab}$ & & & & $\mathrm{X}$ \\
\hline Eugenia brevistyla D.Legrand & 1328 & & $\mathrm{ab}$ & & & & $\mathrm{X}$ \\
\hline Eugenia catharinae O. Berg & 1165 & guamirim & $\mathrm{ab}$ & & & $\mathrm{X}$ & \\
\hline Eugenia multicostata D.Legrand & 1234 & pau-alazão & $\mathrm{ab}$ & & $\mathrm{X}$ & & \\
\hline Eugenia nutans O.Berg & 1330 & camboim & $a b$ & & & & $\mathrm{X}$ \\
\hline Eugenia stigmatosa DC. & 1261 & & $a b$ & & & $\mathrm{X}$ & $\mathrm{X}$ \\
\hline Eugenia sulcata Spring ex Mart. & 1154 & batinga & av & & & & $\mathrm{X}$ \\
\hline Eugenia uniflora $\mathrm{L}$. & 1332 & pitanga & $a b$ & & & & $\mathrm{X}$ \\
\hline Marlierea excoriata Mart. & 1310 & & av & & & & $\mathrm{X}$ \\
\hline Marlierea reitzii D. Legrand & 1162 & guamirim-fueiro & av & & & & $\mathrm{X}$ \\
\hline Marlierea tomentosa Cambess. & 1309 & & av & & & & $\mathrm{X}$ \\
\hline Myrcia brasiliensis Kiaersk. & 1113 & & av & & & $\mathrm{X}$ & \\
\hline
\end{tabular}


Tabela 1 (continuação)

\begin{tabular}{|c|c|c|c|c|c|c|c|}
\hline \multirow{2}{*}{ Família / Espécie } & \multirow{2}{*}{$\mathrm{N}^{\circ} \mathrm{Col}$. } & \multirow{2}{*}{ Nome popular } & \multirow[t]{2}{*}{$\mathrm{Fv}$} & \multicolumn{4}{|c|}{ Formações } \\
\hline & & & & $\mathrm{Rh}$ & $\mathrm{Ra}$ & Raa & $\mathrm{Ft}$ \\
\hline Myrcia ilheosensis Kiaersk. & 1120 & & at & & & & $\mathrm{X}$ \\
\hline Myrcia palustris DC. & 1132 & guamirim & at & & & $\mathrm{X}$ & \\
\hline Myrcia pulchra (O.Berg) Kiaersk. & 1060 & & $a b$ & & $\mathrm{X}$ & $\mathrm{X}$ & \\
\hline Myrcia racemosa (O.Berg) Kiaersk. & 1199 & & at & & & & $\mathrm{X}$ \\
\hline Myrcia selloi (Spreng.) N. Silveira & 1313 & cambuí & at & & & & $\mathrm{X}$ \\
\hline Myrcia tijucensis Kiaersk. & 1262 & & $\mathrm{ab}$ & & & $\mathrm{X}$ & $\mathrm{X}$ \\
\hline Myrcianthes gigantea (D.Legrand) D.Legrand & 1324 & pau-pelado & av & & & & $\mathrm{X}$ \\
\hline Psidium cattleianum Sabine & 1076 & araçá & $a b$ & & $\mathrm{X}$ & $\mathrm{X}$ & $\mathrm{X}$ \\
\hline \multicolumn{8}{|l|}{ Nyctaginaceae } \\
\hline Guapira hirsuta (Choisy) Lundell & 1322 & maria-mole & $a b$ & & & & $\mathrm{X}$ \\
\hline Guapira opposita (Vell.) Reitz & 1098 & maria-mole & $a b$ & & $\mathrm{X}$ & $\mathrm{X}$ & $\mathrm{X}$ \\
\hline \multicolumn{8}{|l|}{ Ochnaceae } \\
\hline Ouratea salicifolia (A.St.-Hil. \& Tul.) Engl. & 1161 & & av & & & $\mathrm{X}$ & \\
\hline \multicolumn{8}{|l|}{ Olacaceae } \\
\hline Heisteria silvianii Schwacke & 1315 & casca-de-tatu & $a b$ & & & & $\mathrm{X}$ \\
\hline \multicolumn{8}{|l|}{ Onagraceae } \\
\hline $\begin{array}{l}\text { Ludwigia multinervia (Hook. \& Arn.) } \\
\text { Ramamoorthy }\end{array}$ & 1235 & cruz-de-malta & $a b$ & & $\mathrm{X}$ & & \\
\hline \multicolumn{8}{|l|}{ Orchidaceae } \\
\hline Cyrtopodium flavum Link \& Otto ex Rchb.f. & 1236 & & $\mathrm{e}$ & & $\mathrm{X}$ & $\mathrm{X}$ & $\mathrm{X}$ \\
\hline Epidendrum fulgens Brongn. & 1019 & orquídea-da-praia & $\mathrm{e}$ & $\mathrm{X}$ & $\mathrm{X}$ & & \\
\hline Liparis nervosa (Thunb.) Lindl. & 1331 & orquídea & e & & & & $\mathrm{X}$ \\
\hline Mesadenella cuspidata (Lindl.) Garay & 1262 & orquídea & $\mathrm{e}$ & & & $\mathrm{X}$ & $\mathrm{X}$ \\
\hline Vanilla chamissonis Klotzsch & 1193 & baunilha & $\mathrm{e}$ & & & $\mathrm{X}$ & \\
\hline \multicolumn{8}{|l|}{ Orobanchaceae } \\
\hline Agalinis communis (Cham. \& Schltdl.) D’Arcy & 1066 & & e & $\mathrm{X}$ & & & \\
\hline \multicolumn{8}{|l|}{ Pentaphylacaceae } \\
\hline Ternstroemia brasiliensis Cambess. & 1074 & pinta-noiva & $a b$ & & & $\mathrm{X}$ & $\mathrm{X}$ \\
\hline \multicolumn{8}{|l|}{ Peraceae } \\
\hline Pera glabrata Poepp. ex Baill. & 1152 & coração-de-bugre & $a b$ & & $\mathrm{X}$ & $\mathrm{X}$ & $\mathrm{X}$ \\
\hline \multicolumn{8}{|l|}{ Phyllanthaceae } \\
\hline Hieronyma alchorneoides Allemão & 1316 & licurana & av & & & & $\mathrm{X}$ \\
\hline Phyllanthus niruri L. & 1321 & erva-pombinha & $\mathrm{e}$ & $\mathrm{X}$ & & & \\
\hline \multicolumn{8}{|l|}{ Piperaceae } \\
\hline Peperomia pereskiaefolia (Jacq.) Kunth & 1044 & & $\mathrm{e}$ & & $\mathrm{X}$ & & \\
\hline Piper hispidum $\mathrm{Sw}$. & 1105 & pimenta-de-macaco & $a b$ & & & $\mathrm{X}$ & $\mathrm{X}$ \\
\hline Piper mosenii C.DC. & 1333 & & $a b$ & & & & $\mathrm{X}$ \\
\hline Piper solmsianum C. DC. & 1341 & & $a b$ & & & & $\mathrm{X}$ \\
\hline Piper umbellatum L. & 1334 & pariparoba & sa & & & & $\mathrm{X}$ \\
\hline Piper sp. & 1131 & & $a b$ & & & & $\mathrm{X}$ \\
\hline \multicolumn{8}{|l|}{ Plantaginaceae } \\
\hline $\begin{array}{l}\text { Achetaria ocymoides (Cham. \& Schltdl.) } \\
\text { Wettst. }\end{array}$ & 1094 & bacopa & $\mathrm{e}$ & $\mathrm{X}$ & & & \\
\hline
\end{tabular}


Tabela 1 (continuação)

\begin{tabular}{|c|c|c|c|c|c|c|c|}
\hline \multirow{2}{*}{ Família / Espécie } & \multirow{2}{*}{$\mathrm{N}^{\circ}$ Col. } & \multirow{2}{*}{ Nome popular } & \multirow[t]{2}{*}{$\mathrm{Fv}$} & \multicolumn{4}{|c|}{ Formações } \\
\hline & & & & $\mathrm{Rh}$ & $\mathrm{Ra}$ & Raa & $\mathrm{Ft}$ \\
\hline Plantago tomentosa Lam. & 1009 & tansagem & $\mathrm{e}$ & $\mathrm{X}$ & & & \\
\hline \multicolumn{8}{|l|}{ Poaceae } \\
\hline Andropogon arenarius Hack. & 1042 & capim-da-praia & $\mathrm{e}$ & $\mathrm{X}$ & & & \\
\hline Andropogon bicornis L. & 1051 & capim-rabo-de-burro & $\mathrm{e}$ & $\mathrm{X}$ & & & \\
\hline Cenchrus echinatus $\mathrm{L}$. & 1010 & capim-roseta & $\mathrm{e}$ & $\mathrm{X}$ & & & \\
\hline Eragrostis trichocolea Arechav. & 1122 & capim & $\mathrm{e}$ & $\mathrm{X}$ & & & \\
\hline Eragrostis sp. & 1049 & capim & $\mathrm{e}$ & $\mathrm{X}$ & & & \\
\hline Eustachys retusa (Lag.) Kunth & 1053 & capim & $\mathrm{e}$ & $\mathrm{X}$ & & & \\
\hline Panicum racemosum (P. Beauv.) Spreng. & 1005 & capim-das-dunas & e & $\mathrm{X}$ & & & \\
\hline Panicum sp. & 1216 & capim & e & $\mathrm{X}$ & & & \\
\hline Paspalum pumilum Nees & 1217 & capim & e & $\mathrm{X}$ & & & \\
\hline Paspalum vaginatum $\mathrm{Sw}$. & 1039 & arame-da-praia & $\mathrm{e}$ & $\mathrm{X}$ & & & \\
\hline Spartina ciliata Brongn. & 1104 & capim-da-praia & e & $\mathrm{X}$ & & & \\
\hline Sporobolus virginicus (L.) Kunth & 1104 & capim & $\mathrm{e}$ & $\mathrm{X}$ & & & \\
\hline Stenotaphrum secundatum (Walter) Kuntze & 1050 & grama-santo-agostinho & e & $\mathrm{X}$ & & & \\
\hline Podocarpaceae & & & & & $\mathrm{X}$ & & \\
\hline Podocarpus sellowii Klotzsch ex Endl. & 1318 & pinheiro-bravo & av & & & & $\mathrm{X}$ \\
\hline \multicolumn{8}{|l|}{ Polygalaceae } \\
\hline Polygala cyparissias A.St.-Hil. \& Moq. & 1036 & gelol-da-praia & $\mathrm{e}$ & $\mathrm{X}$ & & & \\
\hline Polygala paniculata $\mathrm{L}$. & 1218 & barba-de-são-joão & $\mathrm{e}$ & $\mathrm{X}$ & & & \\
\hline \multicolumn{8}{|l|}{ Portulacaceae } \\
\hline Portulaca oleracea L. & 1031 & capanga & e & $\mathrm{X}$ & & & \\
\hline \multicolumn{8}{|l|}{ Primulaceae } \\
\hline Myrsine gardneriana A. DC. & 1263 & capororoca & $a b$ & & & & \\
\hline Myrsine guianensis (Aubl.) Kuntze & 1099 & capororoca & $\mathrm{ab}$ & & & $\mathrm{X}$ & \\
\hline Myrsine parvifolia A. DC. & 1145 & capororoca & $a b$ & & $\mathrm{X}$ & $\mathrm{X}$ & \\
\hline Myrsine rubra M.F.Freitas \& Kin.-Gouv. & 1339 & capororoca & $\mathrm{ab}$ & & $\mathrm{X}$ & & $\mathrm{X}$ \\
\hline Myrsine umbellata Mart. & 1064 & capororoca & av & & & & \\
\hline Myrsine venosa A.DC. & 1129 & capororoca & av & & $\mathrm{X}$ & $\mathrm{X}$ & $\mathrm{X}$ \\
\hline Rosaceae & & & & & $\mathrm{X}$ & $\mathrm{X}$ & \\
\hline Prunus myrtifolia (L.) Urb. & 1362 & pessegueiro-bravo & av & & & & \\
\hline Rubiaceae & & & & & & $\mathrm{X}$ & \\
\hline Amaioua guianensis Aubl. & 1323 & carvoeiro & at & & & & $\mathrm{X}$ \\
\hline $\begin{array}{l}\text { Amaioua intermedia Mart. ex Schult. \& Schult. } \\
\text { F. }\end{array}$ & 1204 & & at & & & & \\
\hline Bathysa australis (A. St.-Hil.) K. Schum. & 1329 & macuqueiro & av & & & $\mathrm{X}$ & $\mathrm{X}$ \\
\hline Chiococca alba (L.) Hitchc. & 1062 & purga-preta & $\mathrm{ab}$ & & & & $\mathrm{X}$ \\
\hline Coccocypselum condalia Pers. & 1157 & erva-de-rato & $\mathrm{e}$ & & $\mathrm{X}$ & $\mathrm{X}$ & $\mathrm{X}$ \\
\hline $\begin{array}{l}\text { Coccocypselum lanceolatum (Ruiz \& Pav.) } \\
\text { Pers. }\end{array}$ & 1340 & erva-de-rato & e & & & & $\mathrm{X}$ \\
\hline Cordiera concolor (Cham.) Kuntze & 1347 & marmeladinha & $a b$ & & & & $\mathrm{X}$ \\
\hline $\begin{array}{l}\text { Diodella apiculata (Willd. ex Roem. \& Schult.) } \\
\text { Delprete }\end{array}$ & 1022 & engana-bobo & e & $\mathrm{X}$ & & & \\
\hline
\end{tabular}


Tabela 1 (continuação)

\begin{tabular}{|c|c|c|c|c|c|c|c|}
\hline \multirow{2}{*}{ Família / Espécie } & \multirow{2}{*}{$\mathrm{N}^{\circ}$ Col. } & \multirow{2}{*}{ Nome popular } & \multirow[t]{2}{*}{$\mathrm{Fv}$} & \multicolumn{4}{|c|}{ Formações } \\
\hline & & & & $\mathrm{Rh}$ & $\mathrm{Ra}$ & Raa & $\mathrm{Ft}$ \\
\hline $\begin{array}{l}\text { Diodella radula (Willd. ex Roem. \& Schult.) } \\
\text { Delprete }\end{array}$ & 1023 & erva-de-lagarto & $\mathrm{e}$ & $\mathrm{X}$ & & & \\
\hline $\begin{array}{l}\text { Margaritopsis cymuligera (Müll. Arg.) C. M. } \\
\text { Taylor }\end{array}$ & 1264 & & $a b$ & & & $X$ & \\
\hline Psychotria carthagenensis Jacq. & 1136 & & $a b$ & & & $\mathrm{X}$ & $\mathrm{X}$ \\
\hline $\begin{array}{l}\text { Psychotria hoffmannseggiana (Willd. ex } \\
\text { Schult.) Müll. Arg. }\end{array}$ & 1133 & & $a b$ & & & & $\mathrm{X}$ \\
\hline Psychotria leiocarpa Cham. \& Schltdl. & 1325 & & $a b$ & & & $\mathrm{X}$ & $\mathrm{X}$ \\
\hline Psychotria nemorosa Gardner & 1265 & & $a b$ & & & & $\mathrm{X}$ \\
\hline Psychotria nuda (Cham. \& Schltdl.) Wawra & 1326 & erva-de-rato & $a b$ & & & & $\mathrm{X}$ \\
\hline Psychotria suterella Müll. Arg. & 1338 & & $a b$ & & & & $\mathrm{X}$ \\
\hline Richardia brasiliensis Gomes & 1169 & poaia & $\mathrm{e}$ & $\mathrm{X}$ & $\mathrm{X}$ & & \\
\hline Rudgea coriacea (Spreng.) K.Schum. & 1155 & & av & & & & $\mathrm{X}$ \\
\hline \multicolumn{8}{|l|}{ Rutaceae } \\
\hline Esenbeckia grandiflora Mart. & 1342 & pau-de-cutia & av & & & & $\mathrm{X}$ \\
\hline \multicolumn{8}{|l|}{ Salicaceae } \\
\hline Casearia sylvestris $\mathrm{Sw}$. & 1168 & cafezeiro & av & & & $\mathrm{X}$ & $\mathrm{X}$ \\
\hline \multicolumn{8}{|l|}{ Sapindaceae } \\
\hline Cupania oblongifolia Mart. & 1348 & camboatá & av & & & & $\mathrm{X}$ \\
\hline Dodonaea viscosa Jacq. & 1054 & vassoura-vermelha & av & $\mathrm{X}$ & $\mathrm{X}$ & $\mathrm{X}$ & $\mathrm{X}$ \\
\hline Matayba guianensis Aubl. & 1201 & camboatá-branco & av & & & & $\mathrm{X}$ \\
\hline Matayba intermedia Radlk. & 1363 & & av & & & & $\mathrm{X}$ \\
\hline Paullinia trigonia Vell. & 1266 & tingui-cipó & 1 & & & $\mathrm{X}$ & $\mathrm{X}$ \\
\hline \multicolumn{8}{|l|}{ Sapotaceae } \\
\hline $\begin{array}{l}\text { Chrysophyllum gonocarpum (Mart. \& Eichler } \\
\text { ex Miq.) Engl. }\end{array}$ & 1343 & mata-olho & av & & & & $\mathrm{X}$ \\
\hline Chrysophyllum inornatum Mart. & 1349 & & av & & & & $\mathrm{X}$ \\
\hline Manilkara subsericea (Mart.) Dubard. & 1267 & maçaranduba & av & & & $\mathrm{X}$ & \\
\hline $\begin{array}{l}\text { Pouteria beaurepairei (Glaz. \& Raunk.) } \\
\text { Baehni }\end{array}$ & 1114 & batatás & av & & & $\mathrm{X}$ & \\
\hline Pouteria venosa (Mart.) Baehni & 1344 & & av & & & & $\mathrm{X}$ \\
\hline \multicolumn{8}{|l|}{ Simaroubaceae } \\
\hline Picramnia parvifolia Engl. & 1268 & cedrinho & av & & & $\mathrm{X}$ & \\
\hline \multicolumn{8}{|l|}{ Smilacaceae } \\
\hline Smilax campestris Griseb. & 1018 & salsaparrilha & e & $\mathrm{X}$ & $\mathrm{X}$ & & \\
\hline \multicolumn{8}{|l|}{ Solanaceae } \\
\hline Solanum inaequale Vell. & 1269 & cuivira & $a b$ & & & $\mathrm{X}$ & $\mathrm{X}$ \\
\hline $\begin{array}{l}\text { Solanum pseudodaphnopsis L.A.Mentz \& } \\
\text { Stehmann }\end{array}$ & 1158 & & $a b$ & & & & $\mathrm{X}$ \\
\hline Solanum pseudoquina A.St.-Hil. & 1138 & quina & $a b$ & & $\mathrm{X}$ & & \\
\hline \multicolumn{8}{|l|}{ Urticaceae } \\
\hline Cecropia pachystachya Trécul & 1345 & embaúba & av & & & & $\mathrm{X}$ \\
\hline Coussapoa microcarpa (Schott) Rizzini & 1270 & figueirinha & av & & & $\mathrm{X}$ & $\mathrm{X}$ \\
\hline \multicolumn{8}{|l|}{ Verbenaceae } \\
\hline Aegiphila fluminensis Vell. & 1346 & & $a b$ & & & & $\mathrm{X}$ \\
\hline Citharexylum myrianthum Cham. & 1337 & tucaneira & av & & & & $\mathrm{X}$ \\
\hline Lantana camara $\mathrm{L}$. & 1067 & camará & sa & & $\mathrm{X}$ & $\mathrm{X}$ & $\mathrm{X}$ \\
\hline
\end{tabular}


As espécies com maior valor de importância em cada formação corroboram os resultados obtidos por outros estudos de estrutura comunitária em restingas do Estado de Santa Catarina de outros Estados da costa brasileira (Assumpção \& Nascimento 2000, Menezes et al. 2012, Korte et al. 2013).

Estrutura Edáfica - O teste de Mantel $(r=0,6 ; p<0,001)$ indicou que há um gradiente edáfico correlacionado com a diversidade e a estrutura das formações de restinga. As variáveis de solo mais relacionadas para explicar as variações nas comunidades foram os teores de matéria orgânica $(\mathrm{MO})$, acidez potencial $(\mathrm{H}+\mathrm{Al})$ e a espessura da serapilheira.

A análise de componentes principais (PCA) mostrou que os dois primeiros componentes explicaram $89 \%$ da variância total dos dados analisados e permitiram a separação das fisionomias de restinga e as suas espécies típicas. As diferenças entre as fisionomias do gradiente de restinga estudado foram mais influenciadas pelas variações nos teores de matéria orgânica $(\mathrm{MO})$, potássio $(\mathrm{K})$, acidez potencial $(\mathrm{H}+\mathrm{Al})$, capacidade de troca cationica

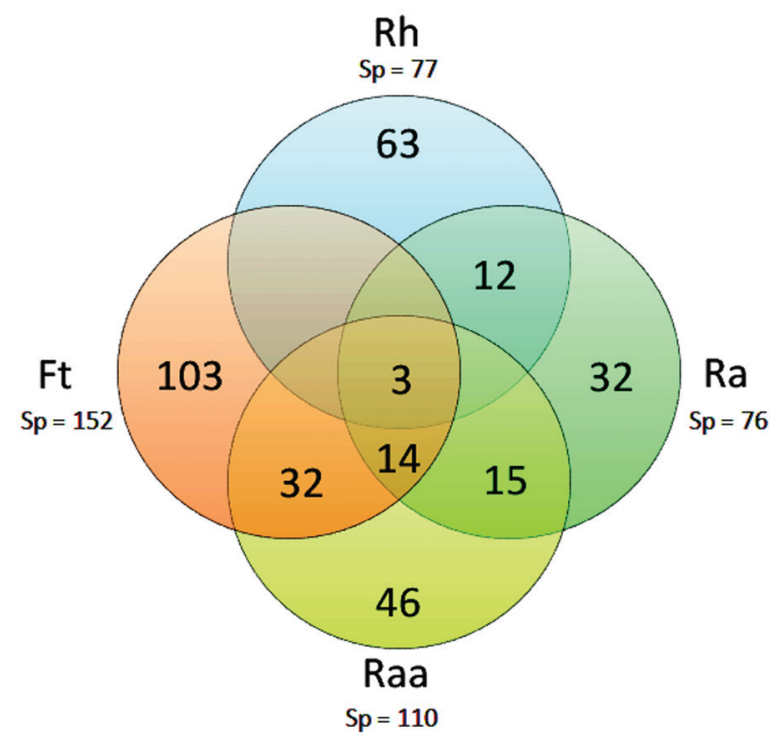

Figura 2. Diagrama de Venn produzido a partir do número de espécies exclusivas e compartilhadas nas formações da restinga do Parque Estadual do Acaraí, São Francisco do Sul, Estado de Santa Catarina, Brasil. Restinga herbácea (Rh), restinga arbustiva (Ra), restinga arbustivo-arbórea (Raa) e floresta de transição $(\mathrm{Ft})$. $\mathrm{Sp}$ : número total de espécies ocorrentes na fisionomia.

Figure 2. Venn diagram produced from the number of unique and shared species in the restinga vegetation of the Parque Estadual do Acaraí, São Francisco do Sul, Santa Catarina State, Brazil. Herb restinga (Rh), Shrub restinga (Ra), Shrub-tree restinga (Raa), and Transition forest (Ft). Sp: total number of species occurring in the vegetation.
(CTC) e espessura da serapilheira, as quais foram positivamente correlacionadas (figura 4, tabela 5). A análise de escalonamento multidimensional não-métrico (NMDS) demonstrou que as formações, apesar de compartilharem algumas espécies, são estruturalmente diferentes entre si $($ stress $=0,034)$ (figura 5).

A análise química dos solos de restinga (tabela 5) mostrou que a acidez do solo aumentou gradativamente do cordão arenoso pós-praia $(\mathrm{Rh})$ em direção à floresta de transição (Ft). Assim, o pH é de início moderadamente ácido e finaliza na floresta como extremamente ácido. Os valores de fósforo $(\mathrm{P})$ e potássio (K) disponíveis foram mais elevados em Ft. $\mathrm{O}$ teor de fósforo $(\mathrm{P})$ mais baixo foi encontrado no solo de Raa e de potássio (K) no solo de Rh. Os teores de alumínio (íons $\mathrm{Al}^{3+}$ em $\mathrm{H}+\mathrm{Al}$ ) foram crescentes ao longo do gradiente. Os valores de sódio $(\mathrm{Na})$ foram muito elevados na Ft, sendo o maior valor obtido para este elemento dentre as formações estudadas. Já o cálcio $(\mathrm{Ca})$ mostrou resultado contrário ao sódio (Na) para todas as fisionomias com exceção da Raa, que exibiu os menores valores de sódio $(\mathrm{Na})$ e cálcio (Ca). Os teores de matéria orgânica (MO) foram crescentes no sentido Rh - Ft. O mesmo padrão ocorreu com a espessura da serapilheira, o que justifica os valores de matéria orgânica (MO) encontrados ao longo do gradiente. Influenciada pelo teor de matéria orgânica (MO), a capacidade de troca cationnica (CTC) foi efetivamente superior na Ft. Quanto à disponibilidade de água nos solos, representada pela umidade gravimétrica, apenas o solo de Ft, de natureza hidromórfica, apresenta aporte hídrico satisfatório quando comparado ao solo das demais formações.

A análise química do solo evidenciou o caráter oligotrófico dos solos das formações de restinga estudadas, conforme o esperado para as restingas brasileiras (Araujo 1987). Exceção se faz ao solo da formação florestal que exibiu comparativamente valores mais elevados para a maior parte dos nutrientes analisados, quando comparado às demais formações. As variações edáficas demonstraram, por meio da PCA, haver diferenças significativas entre as formações de restinga, sendo algumas variáveis mais importantes para essa distinção. Assim, o solo tornou-se um fator chave para a composição florística da vegetação de restinga (Silva 1990), a qual também sofre grande influência pela proximidade do mar e pelo constante déficit hídrico, limitantes, principalmente, à colonização das espécies nas formações imediatas à praia (Daniel 2006), como observado pela menor diversidade na Rh e Ra neste estudo. 


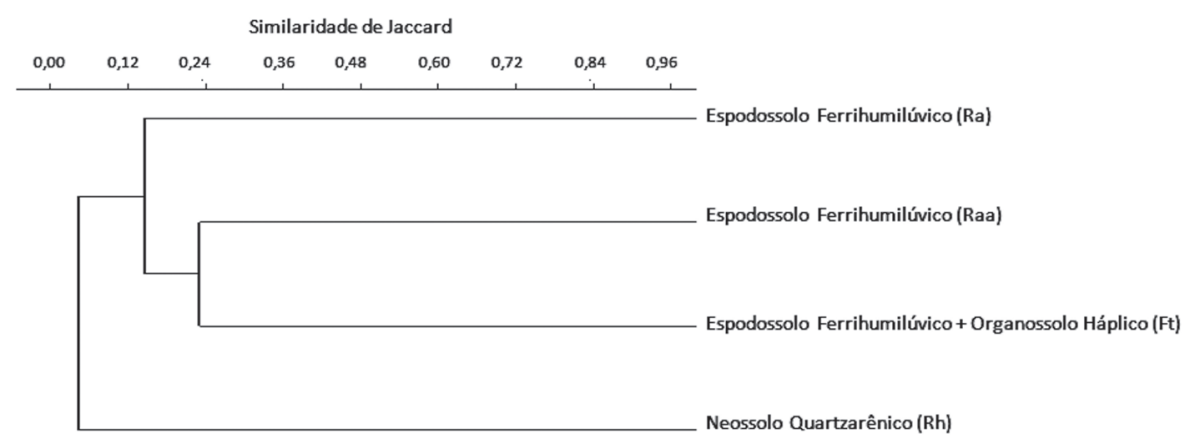

Figura 3. Dendrograma de similaridade florística entre as classes de solo associadas às formações da restinga do Parque Estadual do Acaraí, São Francisco do Sul, Estado de Santa Catarina, Brasil. Restinga herbácea (Rh), restinga arbustiva (Ra), restinga arbustivo-arbórea (Raa) e floresta de transição (Ft).

Figure 3. Dendrogram of floristic similarity between the soil classes associated to the restinga vegetation of the Parque Estadual do Acaraí, São Francisco do Sul, Santa Catarina State, Brazil. Herb restinga (Rh), shrub restinga (Ra), shrub-tree restinga (Raa) and transition forest $(\mathrm{Ft})$.

Tabela 2. Comparação entre as diferentes classes de solo em relação à riqueza de espécies vegetais, famílias mais representativas e índice de diversidade de Shannon $\left(H^{\prime}\right)$ das formações da restinga do Parque Estadual do Acaraí, São Francisco do Sul, SC, Brasil. restinga herbácea (Rh), restinga arbustiva (Ra), restinga arbustivo-arbórea (Raa) e floresta de transição $(\mathrm{Ft}) . H^{\prime}$ : índice de diversidade de Shannon.

Table 2. Comparison of different types of soil in relation to plant species richness, most representative families, and Shannon diversity index $\left(H^{\prime}\right)$ of the restinga vegetation of the Parque Estadual do Acaraí, São Francisco do Sul, Santa Catarina State, Brazil. Herb restinga (Rh), Shrub restinga (Ra), Shrub-tree restinga (Raa), and Transition forest (Ft). H': index of diversity of Shannon.

\begin{tabular}{|c|c|c|c|c|c|c|}
\hline Formação & Classe de solo & № famílias & $\begin{array}{c}\mathrm{N}^{\circ} \\
\text { gêneros }\end{array}$ & No espécies & Famílias (nº espécies) & $H^{\prime}$ \\
\hline $\mathrm{Rh}$ & Neossolo Quartzarênico & 31 & 65 & 77 & $\begin{array}{l}\text { Asteraceae e Poaceae (13), Fabaceae } \\
\text { (7), Amaranthaceae e Cyperaceae (4) }\end{array}$ & 2,89 \\
\hline $\mathrm{Ra}$ & $\begin{array}{l}\text { Espodossolo } \\
\text { Ferrihumilúvico }\end{array}$ & 32 & 62 & 76 & $\begin{array}{l}\text { Asteraceae (16), Fabaceae (9), } \\
\text { Bromeliaceae (5), Primulaceae } \\
\text { (4), Cactaceae, Cyperaceae, } \\
\text { Melastomataceae e Myrtaceae (3) }\end{array}$ & 2,47 \\
\hline Raa & $\begin{array}{l}\text { Espodossolo } \\
\text { Ferrihumilúvico }\end{array}$ & 47 & 81 & 110 & $\begin{array}{l}\text { Asteraceae (13), Fabaceae e } \\
\text { Myrtaceae (9), Bromeliaceae (8), } \\
\text { Moraceae e Rubiaceae (5) }\end{array}$ & 2,76 \\
\hline $\mathrm{Ft}$ & $\begin{array}{c}\text { Espodossolo } \\
\text { Ferrihumilúvico + } \\
\text { Organossolo Háplico }\end{array}$ & 55 & 112 & 152 & $\begin{array}{l}\text { Myrtaceae (16), Rubiaceae (14), } \\
\text { Fabaceae (11), Moraceae (9), } \\
\text { Arecaceae (7) }\end{array}$ & 3,29 \\
\hline
\end{tabular}

$\mathrm{O} \mathrm{pH}$ ácido do solo na área de estudo é tido como um fator que pode afetar o estabelecimento das comunidades vegetais (Santos-Filho et al. 2013) e reduzir as taxas de decomposição no solo (Silva 1990), o que justifica o maior teor de matéria orgânica (MO) obervado no Espodossolo na Ft.
Quanto mais ácido o pH, maior será a concentração de alumínio sob a forma de cátion trocável, o qual pode se tornar tóxico às plantas quando em alta concentração (Silva 1990). A presença de alumínio associada a baixos níveis de cálcio dificulta a absorção de água e a captura de nutrientes pelas plantas, provocando o 
desenvolvimento de um sistema radicial superficial (Casagrande 2003), como percebido na vegetação de todas as fisionomias de restinga estudadas. Além disso, o alumínio é responsável por precipitar o fósforo, tornando-o insolúvel no solo, o que pode acarretar o déficit de fósforo nas plantas (Sutcliffe \& Baker 1989).

Por outro lado, áreas com maior teor de matéria orgânica são mais propensas ao desenvolvimento de plantas lenhosas, cuja fertilidade associada à disponibilidade de água nos solos determina as diferenças florísticas e estrututrais das formações (Silva \& Somner 1984, Sztutman \& Rodrigues 2002, Cestaro \& Soares 2004, Almeida Jr. et al. 2009). Os resultados obtidos pela PCA neste estudo suportam essa hipótese, mostrando a forte influência da matéria orgânica (MO) na organização das comunidades de restinga, apesar da umidade gravimétrica não ter sido responsável pelo arranjo das formações estudadas. Associado a isso, ocorre o aumento progressivo na espessura da serapilheira ao longo do gradiente de restinga em direção $\mathrm{Ft}$, que representa a matéria orgânica a ser decomposta no sistema para liberação de nutrientes disponíveis no solo.

Os teores de matéria orgânica (MO) mostraram relação proporcional com a capacidade de troca catiônica (CTC), sendo os maiores valores na Ft sobre

Tabela 3. Espécies amostradas na formação de restinga herbácea do Parque Estadual do Acaraí, São Francisco do Sul, Estado de Santa Catarina, Brasil, em ordem decrescente de VC. Número de indivíduos (Ni), freqüência absoluta (FA \%), freqüência relativa (FR \%), cobertura absoluta (CA \%), cobertura relativa (CR \%), valor de cobertura (VC \%), valor indicador (Indv \%) e nível de significância do valor indicador $(p)$.

Table 3. Species sampled in the herb restinga vegetation of the Parque Estadual do Acaraí, São Francisco do Sul, Santa Catarina State, Brazil, in descending order of VC. Number of individuals (Ni), absolute frequency (FA \%), relative frequency (FR \%), absolute coverage (CA \%), relative coverage (CR \%), coverage value (VC \%), indicator value (Indv \%), and level of significance of the indicator value $(p)$.

\begin{tabular}{lcrrrrrrrc}
\hline Família & Espécie & Ni & FA & FR & CA & CR & VC & Indv & $p$ \\
\hline Smilacaceae & Smilax campestris & 14 & 93,3 & 10,6 & 17,0 & 7,8 & 18,4 & 93 & 0,001 \\
Goodeniaceae & Scaevola plumieri & 8 & 53,3 & 6,1 & 21,9 & 10,1 & 16,2 & 53 & 0,001 \\
Fabaceae & Canavalia rosea & 9 & 60,0 & 6,8 & 18,4 & 8,5 & 15,3 & 60 & 0,001 \\
Fabaceae & Stylosanthes viscosa & 7 & 46,7 & 5,3 & 20,7 & 9,5 & 14,8 & 47 & 0,002 \\
Amaranthaceae & Blutaparon portulacoides & 10 & 66,7 & 7,6 & 15,3 & 7,1 & 14,6 & 67 & 0,001 \\
Convolvulaceae & Ipomoea imperati & 14 & 93,3 & 10,6 & 6,5 & 3,0 & 13,6 & 93 & 0,001 \\
Cyperaceae & Remirea maritima & 13 & 86,7 & 9,8 & 6,3 & 2,9 & 12,8 & 87 & 0,001 \\
Convolvulaceae & Ipomoea pes-caprae & 7 & 46,7 & 5,3 & 14,3 & 6,6 & 11,9 & 50 & 0,001 \\
Apocynaceae & Oxypetalum tomentosum & 8 & 53,3 & 6,1 & 10,4 & 4,8 & 10,9 & 53 & 0,001 \\
Calyceraceae & Acicarpha bonariensis & 1 & 6,7 & 0,8 & 20,0 & 9,2 & 10,0 & 67 & 0,001 \\
Asteraceae & Symphyopappus casarettoi & 1 & 6,7 & 0,8 & 20,0 & 9,2 & 10,0 & 67 & 0,001 \\
Calyceraceae & Calycera crassifolia & 8 & 53,3 & 6,1 & 4,9 & 2,2 & 8,3 & 53 & 0,001 \\
Poaceae & Paspalum vaginatum & 6 & 40,0 & 4,5 & 6,5 & 3,0 & 7,5 & 40 & 0,001 \\
Poaceae & Spartina ciliata & 5 & 33,3 & 3,8 & 3,4 & 1,6 & 5,4 & 33 & 0,001 \\
Apiaceae & Hydrocotyle bonariensis & 5 & 33,3 & 3,8 & 2,5 & 1,2 & 4,9 & 33 & 0,001 \\
Malvaceae & Pavonia alnifolia & 2 & 13,3 & 1,5 & 6,3 & 2,9 & 4,4 & 13 & 0,002 \\
Asteraceae & Gamochaeta americana & 3 & 20,0 & 2,3 & 2,5 & 1,2 & 3,4 & 20 & 0,003 \\
Portulacaceae & Portulaca oleracea & 3 & 20,0 & 2,3 & 2,5 & 1,2 & 3,4 & 20 & 0,002 \\
Fabaceae & Desmodium adscendens & 2 & 13,3 & 1,5 & 2,5 & 1,2 & 2,7 & 13 & 0,040 \\
Apiaceae & Centela asiatica & 1 & 6,7 & 0,8 & 2,5 & 1,2 & 1,9 & 6 & 0,050 \\
Fabaceae & Centrosema virginianum & 1 & 6,7 & 0,8 & 2,5 & 1,2 & 1,9 & 6 & 0,200 \\
Euphorbiaceae & Euphorbia hyssopifolia & 1 & 6,7 & 0,8 & 2,5 & 1,2 & 1,9 & 6 & 1,000 \\
Asteraceae & Pterocaulon purpurascens & 1 & 6,7 & 0,8 & 2,5 & 1,2 & 1,9 & 6 & 1,000 \\
Malvaceae & Sida carpinifolia & 1 & 6,7 & 0,8 & 2,5 & 1,2 & 1,9 & 6 & 1,000 \\
Poaceae & Stenotaphrum secundatum & 1 & 6,7 & 0,8 & 2,5 & 1,2 & 1,9 & 6 & 1,000 \\
\hline
\end{tabular}


Tabela 4. Espécies amostradas nas formações de restinga arbustiva, restinga arbustivo-arbórea e floresta de transição do Parque Estadual do Acaraí, São Francisco do Sul, Estado de Santa Catarina, Brasil, em ordem decrescente de VI. Número de indivíduos (Ni), freqüência absoluta (FA \%), freqüência relativa (FR \%), densidade absoluta (DA ind ha ${ }^{-1}$ ), densidade relativa (DR \%), dominância absoluta (DoA m² ha ${ }^{-1}$ ), dominância relativa (DoR \%), valor de importância (VI), valor indicador (Indv \%) e nível de significância do valor indicador $(p)$.

Table 4. Species sampled in the Shrub restinga (Ra), Shrub-tree restinga (Raa), and Transition forest (Ft) vegetation of the Parque Estadual do Acaraí, São Francisco do Sul, Santa Catarina State, Brazil, in descending order of VI. Number of individuals (Ni), absolute frequency (FA \%), relative frequency (FR \%), absolute density (DA ind ha ${ }^{-1}$ ), relative density (DR $\%$ ), absolute dominance (DoA $\mathrm{m}^{2} \mathrm{ha}^{-1}$ ), relative dominance (DoR \%), importance value (VI), indicator value (Indv \%), and level of significance of the indicator value $(p)$.

\begin{tabular}{|c|c|c|c|c|c|c|c|c|c|c|c|}
\hline Família & Espécie & $\mathrm{Ni}$ & FA & FR & DA & DR & DoA & DoR & VI & Indv & $p$ \\
\hline \multicolumn{12}{|l|}{ Restinga arbustiva } \\
\hline Nyctaginaceae & Guapira opposita & 138 & 93,3 & 10,8 & 920,0 & 28,8 & 1,034 & 38,82 & 78,3 & 100 & 0,001 \\
\hline Myrtaceae & Myrcia pulchra & 69 & 86,7 & 10,0 & 460,0 & 14,4 & 0,297 & 11,16 & 35,5 & 87 & 0,001 \\
\hline Marcgraviaceae & Norantea brasiliensis & 41 & 60,0 & 6,9 & 273,3 & 8,5 & 0,334 & 12,55 & 28,0 & 80 & 0,001 \\
\hline Clusiaceae & Clusia criuva & 18 & 93,3 & 10,8 & 120,0 & 3,8 & 0,200 & 7,49 & 22,0 & 40 & 0,002 \\
\hline Myrtaceae & Psidium cattleianum & 31 & 46,7 & 5,4 & 206,7 & 6,5 & 0,180 & 6,76 & 18,6 & 46 & 0,002 \\
\hline Anacardiaceae & Schinus terebinthifolius & 28 & 80,0 & 9,2 & 186,7 & 5,8 & 0,064 & 2,41 & 17,5 & 80 & 0,001 \\
\hline Boraginaceae & Cordia curassavica & 34 & 66,7 & 7,7 & 226,7 & 7,1 & 0,059 & 2,23 & 17,0 & 67 & 0,001 \\
\hline Primulaceae & Myrsine venosa & 23 & 46,7 & 5,4 & 153,3 & 4,8 & 0,080 & 3,01 & 13,2 & 47 & 0,001 \\
\hline Lauraceae & Ocotea pulchella & 24 & 20,0 & 2,3 & 160,0 & 5,0 & 0,120 & 4,52 & 11,8 & 20 & 0,070 \\
\hline Fabaceae & Lonchocarpus sericeus & 13 & 40,0 & 4,6 & 86,7 & 2,7 & 0,027 & 1,02 & 8,3 & 20 & 0,001 \\
\hline Peraceae & Pera glabrata & 10 & 33,3 & 3,8 & 66,7 & 2,1 & 0,063 & 2,36 & 8,3 & 33 & 0,005 \\
\hline Asteraceae & Symphyopappus casarettoi & 7 & 26,7 & 3,1 & 46,7 & 1,5 & 0,020 & 0,76 & 5,3 & 27 & 0,011 \\
\hline Asteraceae & Baccharis sphenophylla & 6 & 13,3 & 1,5 & 40,0 & 1,3 & 0,054 & 2,06 & 4,8 & 13 & 0,200 \\
\hline Rubiaceae & Chiococca alba & 6 & 26,7 & 3,1 & 40,0 & 1,3 & 0,012 & 0,44 & 4,8 & 27 & 0,009 \\
\hline Fabaceae & Sophora tomentosa & 4 & 20,0 & 2,3 & 26,7 & 0,8 & 0,024 & 0,91 & 4,1 & 20 & 0,040 \\
\hline Solanaceae & Solanum pseudoquina & 4 & 20,0 & 2,3 & 26,7 & 0,8 & 0,024 & 0,89 & 4,0 & 20 & 0,050 \\
\hline Clethraceae & Clethra scabra & 5 & 20,0 & 2,3 & 33,3 & 1,0 & 0,010 & 0,36 & 3,7 & 20 & 0,050 \\
\hline Primulaceae & Myrsine parvifolia & 8 & 6,7 & 0,8 & 53,3 & 1,7 & 0,029 & 1,09 & 3,5 & 6 & 1,000 \\
\hline Aquifoliaceae & Ilex theezans & 4 & 20,0 & 2,3 & 26,7 & 0,8 & 0,010 & 0,38 & 3,5 & 20 & 0,040 \\
\hline Fabaceae & Senna pendula & 2 & 13,3 & 1,5 & 13,3 & 0,4 & 0,006 & 0,21 & 2,2 & 13 & 0,300 \\
\hline Myrtaceae & Eugenia multicostata & 1 & 6,7 & 0,8 & 6,7 & 0,2 & 0,008 & 0,29 & 1,3 & 6 & 1,000 \\
\hline Erythroxylaceae & Erythroxylum cuspidifolium & 1 & 6,7 & 0,8 & 6,7 & 0,2 & 0,003 & 0,13 & 1,1 & 6 & 1,000 \\
\hline Melastomataceae & Tibouchina clavata & 1 & 6,7 & 0,8 & 6,7 & 0,2 & 0,002 & 0,07 & 1,0 & 6 & 1,000 \\
\hline Asteraceae & Baccharis singularis & 1 & 6,7 & 0,8 & 6,7 & 0,2 & 0,002 & 0,07 & 1,0 & 6 & 1,000 \\
\hline Primulaceae & Myrsine guianensis & 1 & 6,7 & 0,8 & 6,7 & 0,2 & 0,001 & 0,05 & 1,0 & 6 & 1,000 \\
\hline \multicolumn{12}{|c|}{ Restinga arbustivo-arbórea } \\
\hline $\begin{array}{l}\text { Lauraceae } \\
\text { "continua" }\end{array}$ & Ocotea pulchella & 95 & 93,3 & 7,0 & 633,3 & 13,8 & 4,90 & 19,98 & 40,7 & 93 & 0,001 \\
\hline Peraceae & Pera glabrata & 110 & 100 & 7,5 & 733,3 & 15,9 & 4,05 & 16,49 & 39,9 & 100 & 0,001 \\
\hline Pentaphylacaceae & Ternstroemia brasiliensis & 83 & 93,3 & 7,0 & 553,3 & 12 & 4,20 & 17,13 & 36,1 & 93 & 0,001 \\
\hline Primulaceae & Myrsine venosa & 81 & 86,7 & 6,5 & 540,0 & 11,7 & 2,78 & 11,34 & 29,5 & 87 & 0,001 \\
\hline Aquifoliaceae & Ilex theezans & 80 & 93,3 & 7,0 & 533,3 & 11,6 & 2,55 & 10,38 & 28,9 & 93 & 0,001 \\
\hline Clusiaceae & Clusia criuva & 39 & 80,0 & 6,0 & 260,0 & 5,7 & 2,59 & 10,56 & 22,2 & 80 & 0,001 \\
\hline Myrtaceae & Psidium cattleianum & 43 & 86,7 & 6,5 & 286,7 & 6,2 & 0,59 & 2,39 & 15,1 & 87 & 0,001 \\
\hline
\end{tabular}


Tabela 4 (continuação)

\begin{tabular}{|c|c|c|c|c|c|c|c|c|c|c|c|}
\hline Família & Espécie & $\mathrm{Ni}$ & FA & FR & DA & $\mathrm{DR}$ & DoA & DoR & VI & Indv & $p$ \\
\hline Nyctaginaceae & Guapira opposita & 18 & 46,7 & 3,5 & 120,0 & 2,6 & 0,23 & 0,92 & 7,0 & 73 & 0,001 \\
\hline Fabaceae & Andira anthelmia & 9 & 46,7 & 3,5 & 60,0 & 1,3 & 0,28 & 1,14 & 5,9 & 33 & 0,004 \\
\hline Annonaceae & Guatteria australis & 10 & 40,0 & 3,0 & 66,7 & 1,4 & 0,17 & 0,68 & 5,1 & 20 & 0,050 \\
\hline Rubiaceae & Amaioua intermedia & 10 & 33,3 & 2,5 & 66,7 & 1,4 & 0,13 & 0,52 & 4,5 & 13 & 0,200 \\
\hline Fabaceae & Balizia pedicellaris & 6 & 40,0 & 3,0 & 40,0 & 0,9 & 0,06 & 0,26 & 4,1 & 20 & 0,050 \\
\hline Celastraceae & Maytenus robusta & 8 & 26,7 & 2,0 & 53,3 & 1,2 & 0,22 & 0,88 & 4,0 & 20 & 0,050 \\
\hline Fabaceae & Abarema langsdorffii & 6 & 26,7 & 2,0 & 40,0 & 0,9 & 0,22 & 0,88 & 3,7 & 6 & 1,000 \\
\hline Melastomataceae & Miconia pusilliflora & 7 & 33,3 & 2,5 & 46,7 & 1,0 & 0,04 & 0,15 & 3,7 & 6 & 1,000 \\
\hline Salicaceae & Casearia sylvestris & 6 & 26,7 & 2,0 & 40,0 & 0,9 & 0,18 & 0,72 & 3,6 & 27 & 0,010 \\
\hline Calophyllaceae & Calophyllum brasiliense & 5 & 33,3 & 2,5 & 33,3 & 0,7 & 0,08 & 0,32 & 3,5 & 20 & 0,060 \\
\hline Sapindaceae & Dodonaea viscosa & 6 & 26,7 & 2,0 & 40,0 & 0,9 & 0,06 & 0,25 & 3,1 & 13 & 0,200 \\
\hline Erythroxylaceae & Erythroxylum amplifolium & 6 & 26,7 & 2,0 & 40,0 & 0,9 & 0,05 & 0,22 & 3,1 & 27 & 0,010 \\
\hline Sapotaceae & Pouteria beaurepairei & 5 & 20,0 & 1,5 & 33,3 & 0,7 & 0,18 & 0,73 & 2,9 & 6 & 1,000 \\
\hline Myrtaceae & Myrcia bicarinata & 5 & 26,7 & 2,0 & 33,3 & 0,7 & 0,03 & 0,14 & 2,9 & 27 & 0,007 \\
\hline Aquifoliaceae & Ilex dumosa & 3 & 20,0 & 1,5 & 20,0 & 0,4 & 0,18 & 0,75 & 2,7 & 20 & 0,060 \\
\hline Arecaceae & Geonoma elegans & 5 & 20,0 & 1,5 & 33,3 & 0,7 & 0,01 & 0,05 & 2,3 & 20 & 0,060 \\
\hline Myrtaceae & Myrcia ilheosensis & 4 & 20,0 & 1,5 & 26,7 & 0,6 & 0,04 & 0,15 & 2,2 & 6 & 1,000 \\
\hline Clethraceae & Clethra scabra & 3 & 20,0 & 1,5 & 20,0 & 0,4 & 0,03 & 0,14 & 2,1 & 20 & 0,060 \\
\hline Primulaceae & Myrsine umbellata & 5 & 13,3 & 1,0 & 33,3 & 0,7 & 0,04 & 0,17 & 1,9 & 13 & 0,200 \\
\hline Fabaceae & Inga marginata & 2 & 6,7 & 0,5 & 13,3 & 0,3 & 0,25 & 1,04 & 1,8 & 6 & 1,000 \\
\hline Lauraceae & Nectandra oppositifolia & 3 & 13,3 & 1,0 & 20,0 & 0,4 & 0,08 & 0,33 & 1,8 & 13 & 0,200 \\
\hline Solanaceae & Solanum inaequale & 3 & 13,3 & 1,0 & 20,0 & 0,4 & 0,03 & 0,14 & 1,6 & 6 & 1,000 \\
\hline Meliaceae & Trichilia lepidota & 3 & 13,3 & 1,0 & 20,0 & 0,4 & 0,03 & 0,13 & 1,6 & 6 & 1,000 \\
\hline Primulaceae & Myrsine guianensis & 3 & 13,3 & 1,0 & 20,0 & 0,4 & 0,02 & 0,09 & 1,5 & 13 & 0,200 \\
\hline Erythroxylaceae & Erythroxylum deciduum & 3 & 13,3 & 1,0 & 20,0 & 0,4 & 0,02 & 0,08 & 1,5 & 6 & 1,000 \\
\hline Urticaceae & Coussapoa microcarpa & 2 & 13,3 & 1,0 & 13,3 & 0,3 & 0,04 & 0,15 & 1,4 & 13 & 0,200 \\
\hline Simaroubaceae & Picramnia parvifolia & 2 & 13,3 & 1,0 & 13,3 & 0,3 & 0,02 & 0,06 & 1,3 & 6 & 1,000 \\
\hline Myrtaceae & Eugenia stigmatosa & 2 & 13,3 & 1,0 & 13,3 & 0,3 & 0,01 & 0,05 & 1,3 & 13 & 0,200 \\
\hline Lauraceae & Ocotea pulberula & 3 & 6,7 & 0,5 & 20,0 & 0,4 & 0,04 & 0,15 & 1,1 & 6 & 1,000 \\
\hline Moraceae & Ficus luschnathiana & 1 & 6,7 & 0,5 & 6,7 & 0,1 & 0,03 & 0,14 & 0,8 & 6 & 1,000 \\
\hline Fabaceae & Inga sessilis & 1 & 6,7 & 0,5 & 6,7 & 0,1 & 0,02 & 0,10 & 0,7 & 6 & 1,000 \\
\hline Moraceae & Ficus insipida & 1 & 6,7 & 0,5 & 6,7 & 0,1 & 0,02 & 0,10 & 0,7 & 6 & 1,000 \\
\hline Myrtaceae & Myrcia brasiliensis & 1 & 6,7 & 0,5 & 6,7 & 0,1 & 0,01 & 0,06 & 0,7 & 6 & 1,000 \\
\hline Myrtaceae & Myrcia tijucensis & 1 & 6,7 & 0,5 & 6,7 & 0,1 & 0,01 & 0,03 & 0,7 & 6 & 1,000 \\
\hline Aquifoliaceae & Ilex pseudobuxus & 1 & 6,7 & 0,5 & 6,7 & 0,1 & 0,01 & 0,03 & 0,7 & 6 & 1,000 \\
\hline \multicolumn{12}{|c|}{ Floresta de transição } \\
\hline Calophyllaceae & Calophyllum brasiliense & 58 & 93,3 & 8,4 & 386,7 & 17,3 & 4,87 & 22,04 & 47,7 & 93 & 0,001 \\
\hline Euphorbiaceae & Alchornea triplinervia & 25 & 60,0 & 5,4 & 166,7 & 7,4 & 2,76 & 12,50 & 25,4 & 60 & 0,001 \\
\hline Lauraceae & Nectandra oppositifolia & 18 & 66,7 & 6,0 & 120,0 & 5,4 & 2,91 & 13,17 & 24,6 & 67 & 0,001 \\
\hline Anacardiaceae & Schinus terebinthifolius & 15 & 66,7 & 6,0 & 100,0 & 4,5 & 2,67 & 12,07 & 22,6 & 60 & 0,001 \\
\hline Fabaceae & Andira fraxinifolia & 28 & 60,0 & 5,4 & 186,7 & 8,3 & 1,57 & 7,10 & 20,9 & 60 & 0,001 \\
\hline Fabaceae & Dalbergia frutescens & 27 & 60,0 & 5,4 & 180,0 & 8,0 & 1,41 & 6,41 & 19,9 & 60 & 0,001 \\
\hline Annonaceae & Guatteria australis & 15 & 60,0 & 5,4 & 100,0 & 4,5 & 0,22 & 0,99 & 10,9 & 53 & 0,001 \\
\hline
\end{tabular}


Tabela 4 (continuação)

\begin{tabular}{|c|c|c|c|c|c|c|c|c|c|c|c|}
\hline Família & Espécie & $\mathrm{Ni}$ & FA & FR & $\mathrm{DA}$ & DR & DoA & DoR & VI & Indv & $p$ \\
\hline Meliaceae & Cedrela fissilis & 3 & 6,7 & 0,6 & 20,0 & 0,9 & 1,70 & 7,69 & 9,2 & 6 & 1,000 \\
\hline Lauraceae & Ocotea pulchella & 11 & 40,0 & 3,6 & 73,3 & 3,3 & 0,28 & 1,29 & 8,2 & 40 & 0,001 \\
\hline Clusiaceae & Clusia criuva & 11 & 33,3 & 3,0 & 73,3 & 3,3 & 0,28 & 1,25 & 7,5 & 33 & 0,004 \\
\hline Peraceae & Pera glabrata & 9 & 40,0 & 3,6 & 60,0 & 2,7 & 0,13 & 0,57 & 6,9 & 40 & 0,001 \\
\hline Meliaceae & Guarea macrophylla & 10 & 33,3 & 3,0 & 66,7 & 3,0 & 0,19 & 0,84 & 6,8 & 40 & 0,003 \\
\hline Aquifoliaceae & Ilex theezans & 7 & 33,3 & 3,0 & 46,7 & 2,1 & 0,12 & 0,53 & 5,6 & 33 & 0,005 \\
\hline Primulaceae & Myrsine venosa & 5 & 33,3 & 3,0 & 33,3 & 1,5 & 0,23 & 1,04 & 5,5 & 33 & 0,002 \\
\hline Arecaceae & Syagrus romanzoffiana & 3 & 20,0 & 1,8 & 20,0 & 0,9 & 0,55 & 2,50 & 5,2 & 13 & 0,200 \\
\hline Fabaceae & Inga marginata & 9 & 13,3 & 1,2 & 60,0 & 2,7 & 0,26 & 1,18 & 5,1 & 13 & 0,300 \\
\hline Primulaceae & Myrsine rubra & 6 & 26,7 & 2,4 & 40,0 & 1,8 & 0,08 & 0,35 & 4,5 & 27 & 0,010 \\
\hline Lauraceae & Ocotea aciphylla & 5 & 20,0 & 1,8 & 33,3 & 1,5 & 0,10 & 0,46 & 3,8 & 20 & 0,040 \\
\hline Fabaceae & Inga sessilis & 4 & 20,0 & 1,8 & 26,7 & 1,2 & 0,14 & 0,64 & 3,6 & 20 & 0,050 \\
\hline Sapindaceae & Matayba guianensis & 6 & 6,7 & 0,6 & 40,0 & 1,8 & 0,12 & 0,53 & 2,9 & 6 & 1,000 \\
\hline Urticaceae & Cecropia pachystachia & 2 & 13,3 & 1,2 & 13,3 & 0,6 & 0,19 & 0,86 & 2,7 & 13 & 0,200 \\
\hline Salicaceae & Casearia sylvestris & 5 & 6,7 & 0,6 & 33,3 & 1,5 & 0,12 & 0,54 & 2,6 & 6 & 1,000 \\
\hline Myrtaceae & Psidium cattleianum & 4 & 13,3 & 1,2 & 26,7 & 1,2 & 0,04 & 0,19 & 2,6 & 13 & 0,200 \\
\hline Moraceae & Ficus insipida & 2 & 13,3 & 1,2 & 13,3 & 0,6 & 0,15 & 0,68 & 2,5 & 13 & 0,200 \\
\hline Fabaceae & Schizolobium parahyba & 2 & 13,3 & 1,2 & 13,3 & 0,6 & 0,12 & 0,53 & 2,3 & 13 & 0,200 \\
\hline Myrtaceae & Eugenia sulcata & 3 & 13,3 & 1,2 & 20,0 & 0,9 & 0,05 & 0,21 & 2,3 & 13 & 0,200 \\
\hline Piperaceae & Piper solmsianum & 3 & 13,3 & 1,2 & 20,0 & 0,9 & 0,02 & 0,09 & 2,2 & 6 & 1,000 \\
\hline Celastraceae & Maytenus gonoclada & 2 & 13,3 & 1,2 & 13,3 & 0,6 & 0,03 & 0,13 & 1,9 & 13 & 0,200 \\
\hline Arecaceae & Geonoma elegans & 2 & 13,3 & 1,2 & 13,3 & 0,6 & 0,02 & 0,08 & 1,9 & 13 & 0,200 \\
\hline Myrtaceae & Marlierea tomentosa & 2 & 13,3 & 1,2 & 13,3 & 0,6 & 0,02 & 0,08 & 1,9 & 13 & 0,200 \\
\hline Myrtaceae & Myrcia racemosa & 2 & 13,3 & 1,2 & 13,3 & 0,6 & 0,02 & 0,08 & 1,9 & 13 & 0,200 \\
\hline Rubiaceae & Psychotria carthagenensis & 2 & 13,3 & 1,2 & 13,3 & 0,6 & 0,01 & 0,05 & 1,8 & 13 & 0,200 \\
\hline Sapotaceae & Pouteria venosa & 3 & 6,7 & 0,6 & 20,0 & 0,9 & 0,08 & 0,34 & 1,8 & 6 & 1,000 \\
\hline Arecaceae & Bactris setosa & 3 & 6,7 & 0,6 & 20,0 & 0,9 & 0,02 & 0,10 & 1,6 & 6 & 1,000 \\
\hline Clethraceae & Clethra scabra & 1 & 6,7 & 0,6 & 6,7 & 0,3 & 0,11 & 0,51 & 1,4 & 6 & 1,000 \\
\hline Phyllanthaceae & Hieronyma alchorneoides & 1 & 6,7 & 0,6 & 6,7 & 0,3 & 0,11 & 0,49 & 1,4 & 6 & 1,000 \\
\hline Sapindaceae & Dodonaea viscosa & 2 & 6,7 & 0,6 & 13,3 & 0,6 & 0,03 & 0,15 & 1,3 & 6 & 1,000 \\
\hline Annonaceae & Annona neosericea & 2 & 6,7 & 0,6 & 13,3 & 0,6 & 0,03 & 0,14 & 1,3 & 6 & 1,000 \\
\hline Rubiaceae & Bathysa meridionalis & 1 & 6,7 & 0,6 & 6,7 & 0,3 & 0,06 & 0,29 & 1,2 & 6 & 1,000 \\
\hline Malvaceae & Pseudobombax grandiflorum & 1 & 6,7 & 0,6 & 6,7 & 0,3 & 0,05 & 0,25 & 1,1 & 6 & 1,000 \\
\hline Annonaceae & Annona sylvatica & 1 & 6,7 & 0,6 & 6,7 & 0,3 & 0,03 & 0,13 & 1,0 & 6 & 1,000 \\
\hline Lauraceae & Endlicheria paniculata & 1 & 6,7 & 0,6 & 6,7 & 0,3 & 0,03 & 0,12 & 1,0 & 6 & 1,000 \\
\hline Moraceae & Sorocea bonplandii & 1 & 6,7 & 0,6 & 6,7 & 0,3 & 0,03 & 0,12 & 1,0 & 6 & 1,000 \\
\hline Nyctaginaceae & Guapira opposita & 1 & 6,7 & 0,6 & 6,7 & 0,3 & 0,03 & 0,12 & 1,0 & 6 & 1,000 \\
\hline Myrtaceae & Eugenia stigmatosa & 1 & 6,7 & 0,6 & 6,7 & 0,3 & 0,02 & 0,10 & 1,0 & 6 & 1,000 \\
\hline Rubiaceae & Amaioua guianensis & 1 & 6,7 & 0,6 & 6,7 & 0,3 & 0,02 & 0,09 & 1,0 & 6 & 1,000 \\
\hline Moraceae & Maclura tinctoria & 1 & 6,7 & 0,6 & 6,7 & 0,3 & 0,02 & 0,08 & 1,0 & 6 & 1,000 \\
\hline Apocynaceae & Tabernaemontana catharinensis & 1 & 6,7 & 0,6 & 6,7 & 0,3 & 0,01 & 0,05 & 1,0 & 6 & 1,000 \\
\hline Myrtaceae & Myrcia brasiliensis & 1 & 6,7 & 0,6 & 6,7 & 0,3 & 0,01 & 0,05 & 1,0 & 6 & 1,000 \\
\hline Myrtaceae & Eugenia uniflora & 1 & 6,7 & 0,6 & 6,7 & 0,3 & 0,01 & 0,04 & 0,9 & 6 & 1,000 \\
\hline
\end{tabular}


Tabela 4 (continuação)

\begin{tabular}{llcccccccccc}
\hline Família & Espécie & Ni & FA & FR & DA & DR & DoA & DoR & VI & Indv & $p$ \\
\hline Erythroxylaceae & Erythroxylum amplifolium & 1 & 6,7 & 0,6 & 6,7 & 0,3 & 0,01 & 0,03 & 0,9 & 6 & 1,000 \\
Fabaceae & Senna macranthera & 1 & 6,7 & 0,6 & 6,7 & 0,3 & 0,01 & 0,03 & 0,9 & 6 & 1,000 \\
Sapindaceae & Matayba intermedia & 1 & 6,7 & 0,6 & 6,7 & 0,3 & 0,01 & 0,03 & 0,9 & 6 & 1,000 \\
Aquifoliaceae & Ilex brevicuspis & 1 & 6,7 & 0,6 & 6,7 & 0,3 & 0,01 & 0,03 & 0,9 & 6 & 1,000 \\
Arecaceae & Euterpe edulis & 1 & 6,7 & 0,6 & 6,7 & 0,3 & 0,01 & 0,02 & 0,9 & 6 & 1,000 \\
Myrtaceae & Myrcia ilheosensis & 1 & 6,7 & 0,6 & 6,7 & 0,3 & 0,01 & 0,02 & 0,9 & 6 & 1,000 \\
\hline
\end{tabular}

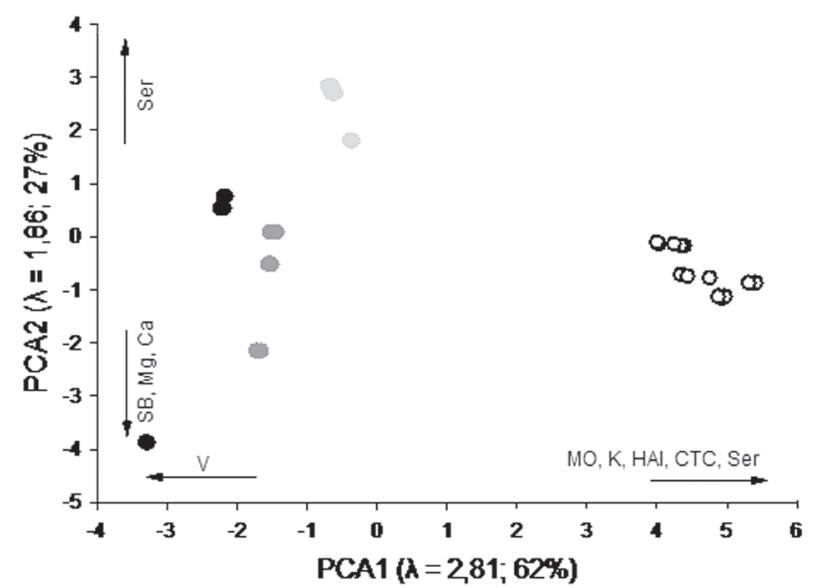

Figura 4. Análise de componentes principais (PCA) mostrando a diferença das formações da restinga do Parque Estadual do Acaraí, São Francisco do Sul, Estado de Santa Catarina, Brasil, em relação às características do solo. As variáveis mais relacionadas com cada componente principal, assim como a explicação e os autovalores dos dois primeiros componentes principais, são mostradas na figura. Restinga herbácea $(\mathrm{Rh})$, restinga arbustiva $(\mathrm{Ra})$, restinga arbustivoarbórea (Raa), floresta de transição $(\mathrm{Ft})$, Comp1 (componente principal 1), Comp 2 (componente principal 2), matéria orgânica $(\mathrm{MO})$, potássio $(\mathrm{K})$, acidez potencial $(\mathrm{H}+\mathrm{Al})$, capacidade de troca catiônica (CTC), espessura da serapilehira (ser), saturação por bases $(\mathrm{V})$, soma de bases (SB), magnésio $(\mathrm{Mg})$ e cálcio $(\mathrm{Ca})$.

Figure 4. Principal component analysis (PCA) showing the difference of the restinga vegetation of the Parque Estadual do Acaraí, São Francisco do Sul, Santa Catarina State, Brazil, in relation to soil characteristics. The variables related to each main component, the eigenvalues, and the explanation of the first two principal components are shown in the figure. Herb restinga (Rh), shrub restinga $(\mathrm{Ra})$, shrub-tree restinga (Raa), transition forest $(\mathrm{Ft})$, Comp1 (principal component 1), Comp 2 (principal component 2), organic matter $(\mathrm{MO})$, potassio $(\mathrm{K})$, potential acidity $(\mathrm{H}+\mathrm{Al})$, ion exchange capacity (CTC), litter thickness (ser), base saturation (V), soma of bases $(\mathrm{SB})$, magnesium $(\mathrm{Mg})$, and calcium $(\mathrm{Ca})$.

Espodossolo, decrescendo abruptamente ao longo do gradiente em direção ao Neossolo Quartzarênico na Rh. Em ambientes de restinga, o teor de matéria orgânica é o principal responsável pelo aumento da capacidade de troca catiônica que, por sua vez, contribui para a retenção de nutrientes no solo (Ruivo et al. 2005, Guedes et al. 2006). Desta forma, a CTC representa a capacidade de liberação de nutrientes, propiciando a manutenção da fertilidade do solo por um período prolongado (Ronquim 2010).

$\mathrm{O}$ teor de potássio $(\mathrm{K})$ também foi crescente ao longo do gradiente e preditor das diferenças entre as formações de restinga. Este nutriente exerce grande efeito sobre o metabolismo das plantas e pode influenciar positivamente a produção de matéria seca (Andrade et al. 2010). Em solos com maior concentração de sódio, como o observado nos solos da restinga estudada, o potássio pode atenuar os efeitos nocivos causados pela salinidade, reduzindo as taxas de transporte de sódio no xilema e sua acumulação nos órgãos aéreos das plantas (Rodrigues et al. 2012).

Resultados semelhantes aos atributos de solo aqui discutidos foram descritos para outras áreas de

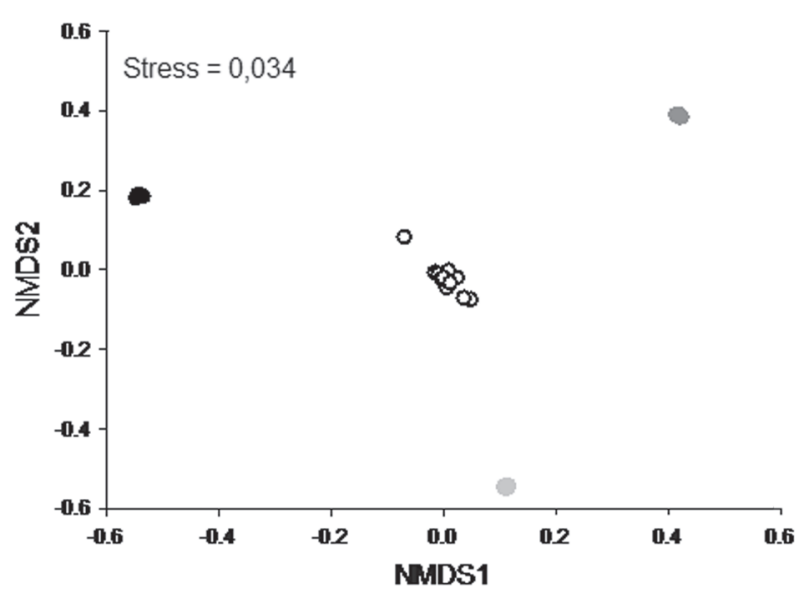

Figura 5. Ordenação NMDS da diferença das comunidades entre as formações da restinga do Parque Estadual do Acaraí, São Francisco do Sul, Estado de Santa Catarina, Brasil. Restinga herbácea (Rh), restinga arbustiva $(\mathrm{Ra})$,restinga arbustivo-arbórea (Raa) e floresta de transição $(\mathrm{Ft})$.

Figure 5. NMDS ordination of the difference among the communities of the restinga vegetation of the Parque Estadual do Acaraí, São Francisco do Sul, Santa Catarina State, Brazil. Herb restinga $(\mathrm{Rh})$, shrub restinga $(\mathrm{Ra})$, shrub-tree restinga (Raa) and transition forest $(\mathrm{Ft})$. 
Tabela 5. Variáveis químicas do solo $(n=3)$, salinidade $(n=3)$, espessura da serapilheira $(n=25)$, umidade gravimétrica $(n=15)$ das classes de solo e formações e correlação entre as variáveis de solo representadas pelos componentes 1 e 2 obtidos pela PCA, da restinga do Parque Estadual do Acaraí, São Francisco do Sul, Estado de Santa Catarina, Brasil. Restinga herbácea $(\mathrm{Rh})$, restinga arbustiva $(\mathrm{Ra})$, restinga arbustivo-arbórea (Raa), floresta de transição $(\mathrm{Ft})$, Comp1 (componente principal 1), Comp 2 (componente principal 2), fósforo $(\mathrm{P})$, potássio $(\mathrm{K})$, sódio $(\mathrm{Na})$, magnésio $(\mathrm{Mg})$, acidez potencial $(\mathrm{H}+$ $\mathrm{Al}$ ), soma de bases (SB), capacidade de troca catiônica (CTC), saturação por bases (V) e matéria orgânica (MO).

Table 5. Soil chemical variables $(\mathrm{n}=3)$, saline $(\mathrm{n}=3)$, accumulation of litter $(\mathrm{n}=25)$, and gravimetric moisture $(\mathrm{n}=15)$ of soil and vegetation types, and correlation between soil variables represented by components 1 and 2 obtained by PCA, in the restinga of the Parque Estadual do Acaraí, São Francisco do Sul, Santa Catarina State, Brazil. Herb restinga (Rh), Shrub restinga $(\mathrm{Ra})$, Shrub-tree restinga (Raa), Transition forest ( $\mathrm{Ft}$ ), Comp1 (principal component 1), Comp 2 (principal component 2), phosphorus $(\mathrm{P})$, potassio $(\mathrm{K})$, sodium $(\mathrm{Na})$, magnesium $(\mathrm{Mg})$, potential acidity $(\mathrm{H}+\mathrm{Al})$, sum of bases $(\mathrm{SB})$, ion exchange capacity (CTC), base saturation (V), and organic matter (MO).

\begin{tabular}{|c|c|c|c|c|c|c|}
\hline \multirow{3}{*}{ Variáveis } & \multicolumn{4}{|c|}{ Classe de Solo (Formação) } & \multicolumn{2}{|c|}{ PCA } \\
\hline & $\begin{array}{c}\text { Neossolo } \\
\text { Quartzarênico }\end{array}$ & $\begin{array}{l}\text { Espodosolo } \\
\text { Ferrihumilúvico }\end{array}$ & $\begin{array}{l}\text { Espodosolo } \\
\text { Ferrihumilúvico }\end{array}$ & $\begin{array}{c}\text { Espodossolo } \\
\text { Ferrihumilúvico }+ \\
\text { Organossolo } \\
\text { Háplico }\end{array}$ & Comp1 & Comp2 \\
\hline & (Rh) & (Ra) & (Raa) & $(\mathrm{Ft})$ & & \\
\hline$\overline{\mathrm{pH}}$ & 5,47 & 4,73 & 3,53 & 3,10 & 0,98 & $-0,19$ \\
\hline $\mathrm{P}\left(\mathrm{mg} \mathrm{dm}^{-3}\right)$ & 2,67 & 2,00 & 1,00 & 25,67 & $-0,82$ & $-0,53$ \\
\hline $\mathrm{K}\left(\right.$ mmolc $\left.\mathrm{dm}^{-3}\right)$ & 1,13 & 1,33 & 1,30 & 2,27 & 0,65 & $-0,27$ \\
\hline $\mathrm{Na}\left(\right.$ mmolc $\left.\mathrm{dm}^{-3}\right)$ & 0,93 & 1,10 & 0,83 & 1,90 & 0,92 & $-0,20$ \\
\hline $\mathrm{Ca}\left(\mathrm{mmolc} \mathrm{dm}^{-3}\right)$ & 9,33 & 10,33 & 5,67 & 7,67 & $-0,31$ & $-0,92$ \\
\hline $\operatorname{Mg}\left(\right.$ mmolc dm $\left.{ }^{-3}\right)$ & 2,67 & 2,33 & 1,00 & 2,00 & $-0,24$ & $-0,95$ \\
\hline $\mathrm{H}+\mathrm{Al} \quad\left(\right.$ mmolc $\left.\mathrm{dm}^{-3}\right)$ & 9,00 & 15,00 & 21,67 & 291,67 & 0,87 & $-0,40$ \\
\hline $\mathrm{SB}\left(\mathrm{mmolc} \mathrm{dm}^{-3}\right)$ & 14,07 & 15,10 & 8,80 & 13,83 & 0,97 & $-0,19$ \\
\hline CTC $\quad\left(\right.$ mmolc $\left.\mathrm{dm}^{-3}\right)$ & 23,07 & 30,10 & 30,47 & 305,50 & $-0,05$ & $-0,99$ \\
\hline V $(\%)$ & 59,67 & 50,00 & 29,00 & 4,33 & 0,96 & $-0,22$ \\
\hline $\mathrm{MO}\left(\mathrm{g} \mathrm{m}^{-3}\right)$ & 7,33 & 14,00 & 14,67 & 82,67 & $-0,94$ & $-0,31$ \\
\hline Serapilheira (cm) & 0,10 & 2,40 & 3,70 & 7,90 & 0,97 & 0,06 \\
\hline Umidade gravimétrica (\%) & 4,90 & 8,30 & 14,10 & 76,20 & 0,71 & $-0,07$ \\
\hline
\end{tabular}

restinga da costa brasileira, indicando, dentre outras variáveis edáficas, a matéria orgânica como fator de grande contribuição na diferenciação da composição de espécies e dos padrões estruturais encontrados (Magnago et al. 2010, Almeida Jr. et al. 2011, SantosFilho et al. 2013).

O conjunto de informações obtidas neste estudo sugere que, dentre as variáveis ambientais aqui consideradas, determinados componentes do solo, com destaque à matéria orgânica $(\mathrm{MO})$ e à espessura da serapilheira, tiveram maior influência sobre a distribuição das espécies e o padrão estrutural das comunidades vegetais, explicando as variações observadas entre as formações.
O conhecimento sobre as influências pedológicas como fatores ambientais condicionantes ao desenvolvimento das comunidades vegetais em ambientes de restinga reforça o pressuposto de que tais comunidades dependem mais da natureza do solo do que do clima. Deste modo, a diversidade florística da planície litorânea, formada por zonas ecológicas estruturalmente complexas e limitada pelos fatores ambientais estressantes, está aclimatada a este ambiente peculiar e se reveste de grande importância biológica. Sob a perspectiva da conservação, torna-se necessário ampliar o esforço de conservação do ambiente de restinga no Estado de Santa Catarina, 
incrementando os estudos em remanescentes ainda não pesquisados como subsídio às políticas de criação de novas áreas para conservação da biodiversidade das restingas.

\section{Agradecimentos}

Este trabalho teve apoio financeiro à coautora, concedido pelo Conselho Nacional de Desenvolvimento Científico e Tecnológico (CNPq), processo n. 309386/2007-1. Agradecemos aos sistematas Anderson Santos de Mello, Rafael Trevisan e Osmar dos Santos Ribas, pelo auxílio na identificação das espécies e ao Prof. André Adrian Padial, pelo suporte nas análises estatísticas.

\section{Literatura citada}

Almeida Jr., E.B. \& Zickel, C.S. 2009. Fisionomia psamófila-reptante: riqueza e composição de espécies na praia da Pipa, Rio Grande do Norte, Brasil. Pesquisas, Botânica 60: 289-299.

Almeida Jr., E.B., Olivo, M.A., Araujo, E.L. \& Zickel, C.S. 2009. Caracterização da vegetação de restinga da RPPN de Maracaípe, Pernambuco, com base na fisionomia, flora, nutrientes do solo e lençol freático. Acta Botanica Brasilica 23: 36-48.

Almeida Jr., E.B., Santos-Filho, F.S., Araújo, E.L. \& Zickel, C.S. 2011. Structural characterization of the woody plants in restinga of Brazil. Journal of Ecology and the Natural Environment 3: 95-103.

Andrade, A.C., Fonseca, D.M., Gomide, J.A., Alvarez, V.H., Martins, C.E. \& Souza, D.P.H. 2010. Produtividade e valor nutritivo do capim-elefante cv. napier sob doses crescentes de nitrogênio e potássio. Revista Brasileira de Zootecnia 29: 1589-1595.

APG III. 2009. An update of the Angiosperm Phylogeny Group classification for the orders and families of flowering plants: APG III. Botanical Journal of the Linnean Society 161: 105-121.

Araujo, D.S.D. 1987. Restingas: síntese dos conhecimentos para a costa sul e sudeste brasileira. In: Anais do $54^{\circ}$ Simpósio sobre Ecossistemas da Costa Sul e Sudeste Brasileira, São Paulo, pp. 333-337.

Araujo, D.S.D. 2000. Análise florística e fitogeografia das restingas do estado do Rio de Janeiro. Tese de Doutorado, Universidade Federal do Rio de Janeiro, Rio de Janeiro.

Araujo, D.S.D. \& Lacerda, L.D. 1987. A natureza das restingas. Ciência Hoje 6: 42-48.

Araujo, D.S.D. \& Henriques, R.P.B. 1984. Análise florística das restingas do estado do Rio de Janeiro. In: L. D. Lacerda et al. (eds.). Restingas: origem, estrutura e processos. CEUFF, Niterói, pp.159-193.
Assis, A.M., Thomaz, L.D. \& Pereira, O.J. 2004. Florística de um trecho de floresta de restinga no município de Guarapari, Espírito Santo, Brasil. Acta Botanica Brasilica 18: 191-201.

Assumpção, J. \& Nascimento, M.T. 2000. Estrutura e composição florística de quatro formações vegetais de restinga no complexo lagunar Grussaí/Iquipari, São João da Barra, RJ, Brasil. Acta Botanica Brasilica 14: 301-315.

Bresolin, A. 1979. Flora da restinga da Ilha de Santa Catarina. Insula 10: 1-55.

Britez, R.M. 2005. Solos. In: M. C. M. Marques \& R. M. Britez (eds.). História natural e conservação da Ilha do Mel, Paraná. UFPR, Curitiba, pp. 49-84.

Britez, R.M., Santos Filho, A., Reissmann, C.B., Silva, S.M., Athayde, S.F., Lima, R.X. \& Quadros, R.M.B. 1997. Nutrientes no solo de duas florestas da planície litorânea da ilha do mel, Paranaguá, PR. Revista Brasileira de Ciências do Solo 21: 625-634.

Casagrande, J.C. 2003. Considerações sobre recuperação da fertilidade do solo para áreas degradadas. In: Anais do Seminário Temático sobre Recuperação de Áreas Degradadas, São Paulo, pp. 92-93.

Castellani, T.T., Folchini, R. \& Scherer, K.Z. 1995. Variação temporal da vegetação em um trecho de baixada úmida entre dunas, Praia da Joaquina, Florianópolis, SC. Insula 24: 37-72.

Causton, D.R. 1988. Introduction to vegetation analysis. Unwin Hyman, London.

Cestaro, L.A. \& Soares, J.J. 2004. Floristic and structural variations, and the phytogeographical relationships of a deciduous forest fragment in Rio Grande do Norte State, Brazil. Acta Botanica Brasilica 18: 203-218.

Chapman, S.B. 1976. Methods in plant ecology. John Wiley \& Sons, New York.

Citadini-Zanette, V., Santos, R. \& Sobral, M. 2001. Levantamento florístico da vegetação arbustiva-arbórea em área ecotonal entre Restinga e Floresta Ombrófila Densa de Terras Baixas (Praia de Palmas, Governador Celso Ramos, Santa Catarina, Brasil). Revista Tecnologia e Ambiente 7: 105-120.

Clarke, K.R. \& Ainsworth, M. 1993. A method of linking multivariate community structure to environmental variables. Marine Ecology Progress Series 92: 205-219.

Cordazzo, C.V. \& Costa, C.S.B. 1989. Associações vegetais das dunas frontais de Garopaba (SC). Ciência e Cultura 41: 906-910.

Costa, C.S.B., Irgang, B.E., Peixoto, A.R. \& Marangoni, J.C. 2003. Composição florística das formações vegetais sobre uma turfeira topotrófica da planície costeira do Rio Grande do Sul, Brasil. Acta Botanica Brasilica 17: 203-212.

Coutinho, C.L. 2006. O conceito de bioma. Acta Botanica Brasilica 20: 13-23. 
Crawley, M.J. 2007. The R book. John Wiley \& Sons, Chichester.

Daniel, R.B. 2006. Florística e fitossociologia da restinga herbáceo-arbustiva do Morro dos Conventos, Araranguá, SC. Dissertação de Mestrado, Universidade do Extremo Sul Catarinense, Criciúma.

Danilevicz, E., Janke, H. \& Pankowski, L.H.S. 1990. Florística e estrutura da comunidade herbácea e arbustiva da praia do Ferrugem, Garopaba, SC. Acta Botanica Brasilica 4: 21-34.

Dantas, T.V.P., Nascimento-Júnior, J.E., Ribeiro, A.S. \& Prata, A.P.N. 2010. Florística e estrutura da vegetação arbustivo-arbórea das Areias Brancas do Parque Nacional Serra de Itabaiana/Sergipe, Brasil. Revista Brasileira de Botânica 33: 575-588.

De'ath G. 1999. Extended dissimilarity: a method of robust estimation of ecological distances from high beta diversity data. Plant Ecology 144: 191-199.

Dorneles, L.P.P. \& Negrelle, R.R.B. 1999. Composição florística e estrutura do compartimento herbáceo de um estágio successional avançado da Floresta Atlântica, no sul do Brasil. Biotemas 12: 7-30.

Dufrene, M. \& Legendre, P. 1997. Species assemblages and indicator species: the need for a flexible asymmetrical approach. Ecological Monographs 67: 345-366.

Eiten, G. 1983. Classificação da vegetação do Brasil. Conselho Nacional de Desenvolvimento Científico e Tecnológico, Brasília.

EMBRAPA. 2013. Sistema brasileiro de classificação de solos. 3 ed. EMBRAPA, Brasília.

EPAGRI. 2002. Mapa de solos: unidade de planejamento regional litoral norte catarinense. Empresa de Pesquisa Agropecuária e Extensão Rural de Santa Catarina, Florianópolis.

Fabris, L.C. 1995. Composição florística e fitossociológica de uma faixa de floresta arenosa litorânea do Parque Estadual de Setiba, Município de Guarapari, ES. Dissertação de Mestrado, Universidade Estadual Paulista, São Paulo.

Falkenberg, D.B. 1999. Aspectos da flora e da vegetação secundária da restinga de Santa Catarina, sul do Brasil. Insula 28: 1-30.

FATMA. 2008. Plano de manejo do Parque Estadual do Acaraí. FATMA, Curitiba.

Felfili, J.M., Roitman, I., Medeiros, M. M.\& Sanchez, M. 2011. Procedimentos e métodos de amostragem da vegetação. In: J. M. Felfili, P.V. Eisenlohr, M.M.R.F. Melo, L. A. Andrade \& J.A.A.M. Neto (eds.). Fitossociologia no Brasil. UFV, Viçosa, pp. 86-121.

Fernandes, A. 2006. Fitogeografia brasileira: províncias florísticas. Realce, Fortaleza.

Fidalgo, O. \& Bononi, L.R. 1989. Técnicas de coleta, preservação e herborização de material botânico. Instituto de Botânica, São Paulo.
Filgueiras, T.S., Nogueira, P.E., Brochado, A.L. \& Guala II, G.F. 1994. Caminhamento: um método expedito para levantamentos florísticos qualitativos. Cadernos de Geociências 12: 39-43.

Gentry, A. 1988. Changes in plant community diversity and floristic composition on environmental and geographical gradients. Annals of the Missouri Botanical Garden 75: 1-34.

Giaretta, A., Menezes, L.F.T. \& Pereira, O.J. 2013. Structure and floristic pattern of a coastal dunes in southeastern Brazil. Acta Botanica Brasilica 27: 87-107.

Gomes, F.H., Vidal-Torrado, P., Macías, F., Gherard, B. \& Perez, X.L.O. 2007. Solos sob vegetação de restinga na ilha do Cardoso (SP), I - caracterização e classificação. Revista Brasileira de Ciências do Solo 31: 1563-1580.

Guedes, D., Barbosa, L.M. \& Martins, S.E. 2006. Composição florística e estrutura fitossociológica de dois fragmentos de floresta de Restinga no Município de Bertioga, SP, Brasil. Acta Botanica Brasilica 20: 299-311.

Guimarães, T.B. 2006. Florística e fenologia reprodutiva de plantas vasculares na restinga do Parque Municipal das Dunas da Lagoa da Conceição, Florianópolis, SC. Dissertação de Mestrado, Universidade Federal de Santa Catarina, Florianópolis.

Hammer, Ø., Harper, D.A.T. \& Ryan, P.D. 2001. Past: paleontological statistics software package for education and data analysis. Palaeontologia Electronica 4: 1-9.

Holzer, W., Crichyno, J. \& Pires, A.C. 2004. Sustentabilidade da urbanização em áreas de restinga: uma proposta de avaliação pós-ocupação. Paisagem Ambiente 19: 49-66.

IBGE. 2012. Manual técnico da vegetação brasileira. 2 ed, IBGE, São Paulo.

Judd, W.S., Campbell, C.S., Kellogg, E.A., Stevens, P.F. \& Donoghue, M.J. 2008. Plant systematics. Sinauer, Sunderland.

Klein, A.S., Citadini-Zanette, V. \& Santos, R. 2007. Florística e estrutura comunitária de restinga herbácea no município de Araranguá, Santa Catarina. Biotemas 20: $15-26$.

Klein, R.M. 1982. A importância sociológica das mirtáceas nas florestas rio grandenses. Silvicultuta 16-A.

Knie, J.L.W. (org.). 2002. Atlas ambiental da região de Joinville: complexo hídrico da Baía da Babitonga. FATMA/GTZ, Florianópolis.

Korte, A., Gasper, A.L., Kruger, A., Sevegnani, L. 2013. Composição florística e estrutura das restingas de Santa Catarina. In: A. C. Vibrans, L. Sevegnani, A. L. Gasper \& D. V. Lingner (eds.). Inventário florísico florestal de Santa Catarina: floresta ombrófila densa. v. 4. Edifurb, Blumenau, pp. 285-309. 
Lista de espécies Flora do Brasil. 2014. Jardim Botânico do Rio de Janeiro. Disponível em: http://floradobrasil. jbrj.gov.br (acesso em 01-II-2014).

Magnago, L.F.S., Martins, S.V., Schaefer, C.E.G.R. \& Neri, A.V. 2010. Gradiente fitofisionômico-edáfico em formações florestais de restinga no sudeste do Brasil. Acta Botanica Brasilica 24: 734-746.

Magurran, A.E. 2013. Medindo a diversidade biológica. UFPR, Curitiba.

Martin, L., Suguio, K., Dominguez, J.M.L., Flexor, J.M. 1997. Geologia do quaternário costeiro do litoral norte do Rio de Janeiro e do Espírito Santo. CPRM/FAPESP, Belo Horizonte.

Matteucci, S.D. \& Colma, A. 1982. Metodologia para el studio de la vegetación. General Secretariat of the Organization of American Status, Washington.

Menezes, C.M., Espinheira, M.J.C.L., Dias, F.J.K. \& Silva, V.I.S. 2012. Composição florística e fitossociologia de trechos da vegetação praial dos litorais norte e sul do estado da Bahia. Revista Biociências 18: 35-41.

Moro, M.F. \& Martins, F.R. 2011. Métodos de levantamento do componente arbóreo-arbustivo. In: J. M. Felfili, P.V. Eisenlohr, M.M.R.F. Melo, L. A. Andrade \& J.A.A.M. Neto (eds.). Fitossociologia no Brasil. UFV, Viçosa, pp. 174-212.

Muller-Dombois, D. \& Ellenberg, H. 1974. Aims and methods of vegetation ecology. J.Wiley and Sons, New York.

Munhoz, C.B.R. \& Araújo, G.M. 2011. Métodos de amostragem do estrato herbáceo-subarbustivo. In: J. M. Felfili, P.V. Eisenlohr, M.M.R.F. Melo, L. A. Andrade \& J.A.A.M. Neto (eds.). Fitossociologia no Brasil. UFV, Viçosa, pp. 213-232.

Negrelle, R.R.B. 1995. Composição florística, estrutura fitossociológica e dinâmica de regeneração da Floresta Atlântica na Reserva Volta Velha, Município de Itapoá, SC. Tese de Doutorado, Universidade Federal de São Carlos, São Carlos.

Negrelle, R.R.B. 2006. Composição florística e estrutura vertical de um trecho de Floresta Ombrófila Densa de planície quaternária. Hoehnea 33: 261-289.

Oliveira, F.A. \& Vieira, C.V. 2008. Proposal of semidetailed geomorphological map of northeastern Santa Catarina State, Brazil. In: Annals of International Meeting and Field Trip Environmental Analysis and Geomorphological Mapping for a Sustainable Development, Roma, pp. 13-15.

Oliveira-Filho, A.T. \& Carvalho, D.A. 1993. Florística e fisionomia da vegetação no extremo norte do litoral da Paraíba. Revista Brasileira de Botânica 16: 115-130.

Pereira, M.C.A., Araujo, D.S.D. \& Pereira, O.J. 2001. Structure of a scrub community of restinga of Barra de Marica - RJ, Rio de Janeiro - Brazil. Revista Brasileira de Botânica 24: 237-281.
PROBIO. 2003. Áreas prioritárias para a conservação, utilização sustentável e repartição de benefícios da biodiversidade brasileira. MMA, Brasília.

Ratter, J.A., Bridgewater, S. \& Ribeiro, J.F. 2003. Analysis of floristic composition of the brazilian cerrado vegetation III: comparison of the woody vegetation of 376 areas. Journal of Botany 60: 57-109.

Reitz, R. 1954. A vegetação de Laguna. Sellowia 6: $243-258$

Reitz, R. 1961. Vegetação da zona marítima de Santa Catarina. Sellowia 13: 17-111.

Rodrigues, C.R.F., Silveira, J.A.G., Silva, E.N., Dutra, A.T.B. \& Viégas, R.A. 2012. Transporte e distribuição de potássio atenuam os efeitos tóxicos do sódio em plantas jovens de pinhão-manso. Revista Brasileira de Ciência do Solo 36: 223-232.

Rogalski, L.D. \& Araújo, A.C. 2005. Flórula da ilha de moleques do sul, Santa Catarina, Brasil. Brazilian Journal of Aquatic Science and Technology 9: 45-48.

Ronquim, C.S. 2010. Conceitos de fertilidade do solo e manejo adequado para as regiões tropicais. EMBRAPA, Campinas.

Ruivo, M.L.P., Amaral, I.G., Faro, M.P.S., Ribeiro, E.L.C., Guedes, A.L.S. \& Santos, M.M.L.S. 2005. Caracterização química da manta orgânica e da matéria orgânica leve em diferentes tipos de solo em uma toposseqüência na ilha de Algodoal/Maiandeua, PA.Boletim do Museu Paraense Emílio Goeldi, Série Ciências Naturais 1: 227-234.

Sá, C.F.C. \& Araujo, D.S.D. 2009. Estrutura e florística de uma floresta de restinga em ipitangas, Saquarema, Rio de Janeiro, Brasil. Rodriguésia 60: 147-170.

Salimon, C.I. \& Negrelle, R.R.B. 2001. Natural regeneration in a quaternary coastal plain in Southern brazilian Atlantic rain forest. Brazilian Archives of Biology and Technology 44: 155-163.

Sampaio, D., Souza, V.C., Oliveira, A.A., Paul-Souza, J. \& Rodrigues, R.R. 2005. Árvores da restinga: guia ilustrado para a identificação de espécies da Ilha do Cardoso. Neotrópica, São Paulo.

Santos, R., Silva, R.C., Pacheco, D., Martins, R. \& Citadini-Zanette, V. 2012. Florística e estrutura do componente arbustivo-arbóreo de mata de restinga arenosa no parque estadual de Itapeva, Rio Grande do Sul. Revista Árvore 36: 1047-1059.

Santos-Filho, F.S., Almeida Jr., E.B. \& Zickel, C.S. 2013. Do edaphic aspects alter vegetation structures in the Brazilian restinga? Acta Botanica Brasilica 27: 613-623.

Scarano, F.R. 2002. Structure, function and floristic relationships of plant communities in stressful Habitats to the brazilian atlantic rainforest. Annals of Botany 90: 517-524.

Scarano, F.R., Duarte, H.M., Ribeiro, K.T., Rodrigues, P.J.F.P., Barcellos, E.M.B., Franco, A.C., Brulfert, J., Deleâens, E. \& Luèttge, U. 2001. Four sites with contrasting environmental stress in southeastern Brazil: relations of species, life form diversity, and geographical distribution to ecophysiological parameters. Botanical Journal of the Linnean Society 136: 345-364. 
Silva, J.G. \& Somner, G.V. 1984. The vegetation of the restinga at Barra de Marica, RJ. In: L. D. Lacerda et al. (eds.). Restingas: origem, estrutura e processos. CEUFF, Niterói, pp. 217-225.

Silva, R.M., Mehlig, U.,Santos, J.U.M.\& Menezes, M.P.M. 2010. The coastal restinga vegetation of Pará, Brazilian Amazon: a synthesis. Revista Brasileira de Botânica 33: 563-573.

Silva, S.M. 1990. Composição floristica e fitossociologia de um trecho de floresta de restinga na ilha do Mel, município de Paranaguá. Dissertação de Mestrado, Universidade Estadual de Campinas, Campinas.

Silva, S.M. 1999. Diagnósticos das restingas do Brasil. In: Anais do Workshop de Avaliação e Ações Prioritárias para Conservação da Biodiversidade da Zona Costeira e Marinha, Porto Seguro, pp. 30.

Silva,S.M. \& Britez, R.M. 2005. A vegetação da planície costeira. In: M. C. M. Marques \& R. M. Britez (eds.). História Natural e Conservação da Ilha do Mel. UFPR, Curitiba, pp. 49-84.

Souza, M.T.R. 2004. O litoral Brasileiro.Revista de Cultura do IMAE 5: 63-67.
Souza, M.L.E.R., Falkenberg, D.B., Amaral, L.G., Fronza, M., Araújo, A.C. \& Sã, M.R. 1992. Vegetação do pontal da Daniela, Florianópolis, SC, Brasil. I.levantamento florístico e mapa fitogeográfico. Insula 21: 87-117.

Sutcliffe, J.F. \& Baker, D.A. 1989. As plantas e os sais minerais. EPU, São Paulo.

Sztutman, M. \& Rodrigues, R.R. 2002. O mosaico vegetacional numa área de floresta contínua da planície litorânea, Parque Estadual de Campina do Encantado, Pariquera-Açu, SP.Revista Brasileira de Botânica 25: 161-176.

Veloso, H.P., Rangel-Filho, A.L.R. \& Lima, J.C.A. 1991. Classificação da vegetação brasileira, adaptada a um sistema universal. IBGE - DERMA, Rio de Janeiro.

Vibrans, A.C., Sevegnani, L., Gasper, A.L., Lingner, D.V. (orgs.). 2012. Inventário florístico florestal de Santa Catarina: diversidade e conservação dos remanescentes florestais. Edifurb, Blumenau. v. 1. 